\title{
Periodicity and Firing Rate As Candidate Neural Codes for the Frequency of Vibrotactile Stimuli
}

\author{
Emilio Salinas, Adrián Hernández, Antonio Zainos, and Ranulfo Romo \\ Instituto de Fisiología Celular, Universidad Nacional Autónoma de México, 04510, México D.F., México
}

\begin{abstract}
The flutter sensation is felt when mechanical vibrations between 5 and $50 \mathrm{~Hz}$ are applied to the skin. Neurons with rapidly adapting properties in the somatosensory system of primates are driven very effectively by periodic flutter stimuli; their evoked spike trains typically have a periodic structure with highly regular time differences between spikes. A long-standing conjecture is that, such periodic structure may underlie a subject's capacity to discriminate the frequencies of periodic vibrotactile stimuli and that, in primary somatosensory areas, stimulus frequency is encoded by the regular time intervals between evoked spikes, not by the mean rate at which these are fired. We examined this hypothesis by analyzing extracellular recordings from primary (S1) and secondary (S2) somatosensory cortices of awake monkeys performing a frequency discrimination task. We quantified stimulus-driven modulations in firing rate and in spike train periodicity, seeking to determine their relevance for frequency dis-
\end{abstract}

crimination. We found that periodicity was extremely high in S1 but almost absent in S2. We also found that periodicity was enhanced when the stimuli were relevant for behavior. However, periodicity did not covary with psychophysical performance in single trials. On the other hand, rate modulations were similar in both areas, and with periodic and aperiodic stimuli, they were enhanced when stimuli were important for behavior, and were significantly correlated with psychophysical performance in single trials. Thus, the exquisitely timed, stimulus-driven spikes of primary somatosensory neurons may or may not contribute to the neural code for flutter frequency, but firing rate seems to be an important component of it.

Key words: awake monkeys; primary somatosensory cortex; secondary somatosensory cortex; neural coding; flutter; discrimination; periodicity; mutual information
The sensation of flutter is produced when mechanical vibrations between 5 and $50 \mathrm{~Hz}$ are applied to the skin (Mountcastle et al., 1967; Talbot et al., 1968). Earlier studies using vibrotactile stimuli reported four basic observations: (1) that sensation in the flutter range is mediated by primary afferent fibers and S1 neurons with rapidly adapting properties associated with Meissner's mechanoreceptors (Mountcastle et al., 1967; Talbot et al., 1968); (2) that these afferents and cortical neurons are driven very effectively by periodic flutter stimuli, which evoke highly periodic spike trains (Mountcastle et al., 1969, 1990; Recanzone et al., 1992); (3) that psychophysical performance in frequency discrimination, which is similar for humans and monkeys (LaMotte and Mountcastle, 1975), correlates closely with the discriminability of the evoked, periodic interspike intervals (Mountcastle et al., 1969, Recanzone et al., 1992); and (4) that in afferent and S1 units, the firing rate, computed over hundreds of milliseconds, changes little within the flutter range (Talbot et al., 1968; Mountcastle et al., 1969, 1990; Recanzone et al., 1992). In view of these results, it was argued that flutter frequency cannot be encoded by the firing rate of rapidly adapting units and that a subject's capacity to discriminate flutter frequencies has to depend on the periodicity of the evoked interspike intervals (Mountcastle et al., 1967, 1969, 1990; Talbot et al., 1968; Recanzone et al., 1992). This led to the proposal that "frequency discrimination is made by a central neural mechanism

Received March 13, 2000; revised April 26, 2000; accepted May 1, 2000.

This research was supported by an International Scholars Award from the Howard Hughes Medical Institute and grants from DGAPA-UNAM and CONACYT to R.R. We thank Bill Newsome and Carlos Brody for invaluable suggestions and discussions, and David Egelman for helpful comments. We appreciate the technical assistance of Sergio Méndez and Federico Jandete. E.S. also thanks Terry Sejnowski and the Howard Hughes Medical Institute for their support during final stages of this work. R.R. designed and performed the experiments with the assistance of A.H. and A.Z.; E.S. designed and performed the data analysis; E.S. and R.R. co-wrote this manuscript.

Correspondence should be addressed to Ranulfo Romo, Instituto de Fisiología Celular, Universidad Nacional Autónoma de México, 04510, México D.F., México. E-mail: rromo@ifisiol.unam.mx.

Dr. Salinas's present address: Computational Neurobiology Laboratory, The Salk Institute, 10010 North Torrey Pines Road, La Jolla, CA 92037.

Copyright (C) 2000 Society for Neuroscience $0270-6474 / 00 / 205503-13 \$ 15.00 / 0$ capable of measuring the lengths of the dominant periodic intervals in the [evoked] trains of impulses" (Mountcastle et al., 1967).

Nevertheless, the fourth and crucial observation was based on a small number of neurons (Mountcastle et al., 1990) or on responses to a narrow range of frequencies applied to anesthetized animals (Recanzone et al., 1992). Additionally, direct microstimulation of Meissner-type primary afferents produced flutter sensations of frequencies that were perceived to increase with evoked firing rate (Ochoa and Torebjörk, 1983). More recently, we also observed that monkeys can discriminate the mean frequencies of aperiodic stimuli, which lack any temporal regularity. Animals can work with aperiodic stimuli whether these are delivered naturally, by a mechanical probe, or artificially, through microinjection of electrical current into S1 (Romo et al., 1998). These considerations cast doubts on the bold proposal quoted above. Is it true, then, that spike periodicity plays a functional role in frequency discrimination and that firing rate does not? If this were the case, cortical somatosensory neurons would provide a solid demonstration of a temporal neural code (Shadlen and Newsome, 1994, 1998; Singer and Gray, 1995; Ahissar, 1998). Here we try to assess the relation, if any, between behavior and stimulus-driven modulations in firing rate and in periodic interspike timing in S1 and S2. The results suggest that firing rate does play an important role in encoding stimulus frequency in our paradigm.

\section{MATERIALS AND METHODS}

Neurophysiology and behavior. The behavioral task is schematized in Figure $1 a$ [see also Romo et al. (1998); Hernández et al. (1997)]. In each trial the monkey had to compare the frequencies of two vibratory stimuli presented consecutively. The sequence of events was as follows. The mechanical probe was lowered, indenting the glabrous (hairless) skin of one digit of the restrained hand; the monkey reacted, placing its free hand on a lever within $1 \mathrm{sec}$ after indentation; after a delay period $(1.5-3 \mathrm{sec})$, the probe oscillated vertically, periodically, at a base frequency; after an interstimulus interval (1-3 sec), a second stimulus was delivered at a comparison frequency; the monkey had to release the lever within 600 msec and press one of two push-buttons to indicate whether the comparison frequency was higher or lower than the base. Both stimuli lasted 500 msec and were delivered to the distal segment of digits 2, 3, or 4 of the left hand via a computer-controlled Chubbuck linear motor stimulator (Chubbuck, 1966), which had a $2 \mathrm{~mm}$ round tip. Initial indentation was $500 \mu \mathrm{m}$. 
Stimulus amplitudes were adjusted to equal subjective intensities (Mountcastle et al., 1990; Hernández et al., 1997). For example, $71 \mu \mathrm{m}$ at $12 \mathrm{~Hz}$ and $51 \mu \mathrm{m}$ at $34 \mathrm{~Hz}(\sim 1.4 \%$ per Hertz). In each trial of the task, a pair of base-comparison frequencies was chosen pseudorandomly from a set typically comprising $\sim 10$ pairs. For one full data collection run, at least five trials per pair had to accumulate with the same stimulus set. Typically, each run included 10 trials per pair. Figure $1, b$ and $c$, shows two stimulus sets commonly used in the experiments (set A was used much more often; see Fig. 1, legend). The numbers inside the grids indicate the percentage of correct discriminations for each pair of frequencies. In set $\mathrm{A}$, the difference between base and comparison was always $8 \mathrm{~Hz}$, and the monkeys performed between 80 and $91 \%$ correct. In set B, smaller and larger differences were combined. In general, monkeys had clear difficulties discriminating when base and comparison frequencies differed by $\sim 2 \mathrm{~Hz}$ or less.

Sinusoidal stimuli were used initially; 137 S1 neurons were studied in this way. Later we switched to trains of short mechanical pulses like those illustrated in Figure 1a. Each of these pulses consisted of a single-cycle sinusoid lasting $20 \mathrm{msec}$. For stimulation at $20 \mathrm{~Hz}, 11$ successive pulses were applied, separated by $50 \mathrm{msec}$. This interval was measured between the beginnings of successive pulses. The data obtained with sinusoidal stimuli were not used in Figure 2 or in comparisons with S2 responses, but they were included in the comparisons between active and passive conditions and between neuronal and psychophysical responses.

Experiments with aperiodic stimuli were also conducted (Romo et al., 1998). In this situation a frequency of $20 \mathrm{~Hz}$ still corresponded to 11 mechanical pulses delivered in a $500 \mathrm{msec}$ period, so the mean interval between pulses was $50 \mathrm{msec}$, but the times between pulses were random. The minimum time between the onsets of consecutive pulses was equal to their width, $20 \mathrm{msec}$, corresponding to a maximum instantaneous frequency of $50 \mathrm{~Hz}$. In practice, then, the total number of pulses delivered was constant across trials of a particular frequency, as in the periodic case, and given the stimulation period of $500 \mathrm{msec}$ and the pulse width of $20 \mathrm{msec}$ those pulses were randomly distributed among $500 / 20=25$ positions in time (except that the first and last pulses were always delivered at the beginning and end of the stimulation interval). The specific tempora pattern of pulses was chosen randomly in each trial, and the patterns were different even for trials with the same mean frequency. The monkeys had to compare the average frequencies of the base and comparison stimuli exactly as before. By average frequency we mean the total number of stimulation pulses divided by the corresponding $500 \mathrm{msec}$ period. These experiments were of two types: with periodic base and aperiodic comparison, or with both aperiodic. Behavioral results from these two variants of the paradigm were pooled. These experiments were performed in blocks interleaved between blocks of regular discrimination with periodic stimuli.

Passive stimulation tests were also performed in blocks and were also interleaved between blocks of active discrimination. During passive stimulation, the hand used to press the push-buttons was restrained, so there were no behavioral reactions, and no reward was provided. Otherwise single trials proceeded as with regular discrimination. The same combinations of base-comparison frequencies were used in experiments with periodic and aperiodic vibrations and in passive and active conditions. However, not all neurons could be recorded long enough to complete all the types of tests, so the numbers of recorded neurons varied across conditions.

Recordings were obtained with an array of seven independent microelectrodes of 2-3 M $\Omega$ (Mountcastle et al., 1990, 1991). Recording procedures were the same as those described by Mountcastle et al. (1990) (see also Romo et al., 1998, 1999). Microelectrodes were aimed at the hand representations in S1 and S2, and the locations of the penetrations were confirmed with standard histological techniques. For the analysis of simultaneously recorded neurons, only pairs with units from different electrodes were used. Separation between microelectrodes was at least $500 \mu \mathrm{m}$ Animals were handled according to the guidelines of the National Institutes of Health and the Society for Neuroscience.

Response measures. The firing rate in each trial was equal to the number of spikes emitted during the $500 \mathrm{msec}$ stimulation period (base or comparison) divided by $0.5 \mathrm{sec}$. The mean firing rate $r$ was obtained by averaging over trials of equal stimulus frequency; therefore, $r$ and its SD $\sigma$ were functions of frequency ( $r$ and $\sigma$ correspond, respectively, to the data points and error bars in Figs. $2 e, 3 e$ ). Response curves of mean firing rate versus stimulus frequency were fitted to linear and Gaussian functions through $\chi^{2}$ minimization (Press et al., 1992). Gaussian tuning curves had four parameters, amplitude $A$, baseline $B$, center frequency $C$, and width $\sigma_{\mathrm{G}}$, such that:

$$
r(s)=B+A \exp \left(-\frac{(s-C)^{2}}{2 \sigma_{G}^{2}}\right),
$$

where $s$ is the stimulus frequency. In the text, angle brackets \langle\rangle indicate averaging over all stimulus frequencies. Thus, $\langle r\rangle$ indicates the mean firing rate averaged over all frequencies. Similarly, $\langle\sigma\rangle$ corresponds to the SD of the firing rate averaged over all frequencies and measures the mean trial-to-trial variability. That is, $r$ and $\sigma$ were first calculated independently for each stimulus frequency and then were averaged across frequencies to obtain $\langle r\rangle$ and $\langle\sigma\rangle$. We also computed a signal-to-noise ratio for each neuron. This was defined as:

$$
S N R=\frac{r_{\max }-r_{\min }}{\langle\sigma\rangle},
$$

where $r_{\max }$ and $r_{\min }$ are the maximum and minimum values, over all stimulus frequencies, of the mean firing rate.

To relate periodicity to performance, we used several measures based on Fourier decompositions of the time signals formed by the evoked trains of spikes. For each trial, the power spectrum of the spike train evoked during the stimulation period (base or comparison) was computed and normalized, having had the DC component removed, so that the total power summed over all positive frequency bins was $100 \%$ (Press et al., 1992). Examples of power spectra for individual trials are shown in Figures $2 b, d$, $3 b, d$. In this way the number of spikes contained in each train had little impact on the resulting Fourier amplitudes, which indicated the proportion of power in the corresponding frequency bins. That is, the Fourier amplitudes were mainly determined by the temporal arrangement of the spikes, not by their number (however, the power spectrum could not be computed in trials in which less than two spikes were emitted). The sampling interval for the spike trains was $0.5 \mathrm{msec}$, and the width of the frequency bins was $1.95 \mathrm{~Hz}$. The latter was limited by the duration of the stimulation period, which for the Fourier analysis we took as $512 \mathrm{msec}$.

Four quantities were extracted from each power spectrum, that is, at each trial. The first two were the power (or amplitude) at the stimulus frequency $\left(P_{\mathrm{S}}\right)$ and the power at twice the stimulus frequency. These two numbers should increase for evoked spikes that are more tightly phaselocked to the stimulation pulses. The third quantity was the maximum power (maximum $y$ coordinate) between 4 and $42 \mathrm{~Hz}$, and its corresponding frequency ( $x$ coordinate) was the fourth quantity, which we denominated the power spectrum frequency at peak (PSFP). The three amplitudes measure how periodic a spike train is, whereas the PSFP is an actual estimate of stimulus frequency that depends on the periodicity of the evoked spike trains; this distinction is crucial (notice that the stimulus frequency needs to be known a priori to compute $P_{\mathrm{S}}$ and the amplitude at twice the stimulus frequency). Note that the resolution of the PSFP depends on the width of the frequency bins of the power spectrum. Consider an example that was relatively common in S1. Suppose a neuron is strongly phaselocked to the stimulus and fires spikes somewhat like a clock, one or two spikes per stimulation pulse, in an approximately periodic fashion. In its spectra, the maximum power would be at the stimulus frequency. Thus, the PSFP amplitude would be equal to $P_{S}$, the PSFP would be the center of the frequency bin nearest to the stimulus frequency, and all three amplitudes (at the PSFP, at the stimulus frequency, and at twice the stimulus frequency) would be much larger than the average power across all bins. Statistical tests applied to any of these four quantities were always performed also with the other three, but sometimes only the results for the most sensitive one are mentioned.

In each trial, we also computed the average interburst interval (AIBI), which measures how often a burst of spikes is produced. A burst was defined as a group of spikes in which all intervals between consecutive spikes were less than $\tau$ msec. Thus, the number of spikes per burst was variable, with a minimum of 1 , and all interspike intervals within a given burst had to be smaller than $\tau$. Large $\tau$ values produced few bursts with many spikes, whereas small $\tau$ values produced many bursts with few or single spikes. Having fixed $\tau$, the AIBI was then computed as the mean value of the time intervals between consecutive burst endings.

Standardized responses. To compare the above responses in correct discrimination trials (hits) versus incorrect discrimination trials (errors), these responses had to be pooled across different base-comparison frequency pairs, because errors were rather infrequent. To illustrate the pooling method, we first consider the firing rate as the response. The standardized rate in a given trial was obtained by taking the original firing rate at that trial, subtracting the mean rate from all trials belonging to the same condition (base-comparison frequency pair), and dividing by the SD from the same subset of trials. The same was done for all trials in the data collection run. This eliminated any differences in response level attributable to preference for one frequency or combination of base and comparison stimulus frequencies-differences across conditions-but left intact any differences within conditions, such as differences between hits and errors. By construction, such a set of standardized rates has zero mean and unit SD. Exactly the same procedure was followed to compute the standardized measures of periodicity, which were the standardized PSFP amplitude, the standardized $P_{\mathrm{S}}$, and the standardized amplitude at twice the stimulus frequency. In all cases the standardized values in individual trials were obtained from the "raw" values by subtracting the mean of the corresponding condition and dividing by the $\mathrm{SD}$, as explained above. Having obtained the standardized responses for each run, trials were separated into two groups. In trials of type 1 , the base frequency was higher than the comparison, and in trials of type 2, the comparison was higher than the base. Thus, for each run and each kind of response, two comparisons between hits and errors were made, one for each set of trials of the same type. In all cases the mean of all standardized responses in error trials was compared with the mean of all standardized responses in hit trials, and the significance of the difference was determined (see Figs. $8,9)$. For each type of comparison, a minimum of five error trials was required. 
Trial-to-trial covariations in the firing rates of simultaneously recorded neurons were measured using Pearson's linear correlation coefficient $\rho$ (Press et al., 1992). This coefficient can be computed easily from the same standardized rates described in the previous paragraph. If $r_{\mathrm{i}}^{\mathrm{a}}$ is the standardized rate of neuron $a$ at trial $i$, the correlation coefficient between neurons $a$ and $b$ is simply:

$$
\rho_{\mathrm{ab}}=\frac{1}{N} \sum_{\mathrm{i}=1}^{\mathrm{N}} r_{\mathrm{i}}^{\mathrm{a}} r_{\mathrm{i}}^{\mathrm{b}},
$$

where $N$ is the total number of trials in the run, including all stimulus frequencies.

Information estimates and other statistics. Having computed the firing rate, the PSFP, and the AIBI in each trial, we quantified how they varied as functions of stimulus frequency for any given cell. For this we used Shannon's mutual information (Cover and Thomas, 1991; Abbott et al., 1996). Information is a measure of association between two quantities, typically stimulus and response. Its magnitude relates to the accuracy with which one of them can be determined given the other. Thus, by computing the information that they provide about a stimulus, two different responses can be compared in the same units, namely, in terms of their capacity to encode the stimulus.

The information that a response $r$ provides about a stimulus $s$ is computed from the probability distributions relating these two variables. In our case, $s$ is the frequency of the applied flutter stimulus. The function $P(r \mid s)$ represents the conditional probability of observing a response $r$ given that the stimulus had a value $s$. The expression $P(r)$ describes the probability of observing a response $r$ regardless of the value of the stimulus, and $P(s)$ is the probability that the stimulus takes a value $s$. When all stimuli are presented the same number of times, $P(s)$ is simply a constant. Using these quantities, the information that the response provides about the stimulus can be computed as:

$$
I=\sum_{\mathrm{r}, \mathrm{s}} P(s) P(r \mid s) \log _{2}\left(\frac{P(r \mid s)}{P(r)}\right) .
$$

Here the sums are over all possible values of the stimulus and the response. Information is measured in bits. If the stimulus $s$ can take $N$ different values, the maximum amount of information that can be provided by any signal is $\log _{2}(N)$ bits. A response carrying these many bits of information lets us determine exactly which of the $N$ stimulus values is presented in any trial; stimulus and response are then maximally correlated. Most information results shown below correspond to experiments in which seven or eight frequencies were applied and in which, therefore, the maximum amount of mutual information was $\log _{2}(8)=3$ bits. An exception to this was made in comparisons between active and passive conditions, because here what mattered was the difference in information values (found with identical numbers of frequencies) across conditions; several sets with 9-11 frequencies were allowed in these cases.

The information that the firing rate provided about the stimulus, $I_{\mathrm{RATE}}$, was computed from the set of firing rates from all trials assuming that the response probability distributions $P(r \mid s)$ were Gaussian (Abbott et al., 1996). (These Gaussians are response probability distributions specific to each stimulus frequency and should not be confused with the Gaussian tuning curves mentioned above.) Then, a correction for finite sampling, based on Monte Carlo methods, was applied [Treves and Panzeri (1995); E. Salinas, unpublished results, but see below]. In practice, this meant that $I$ was first computed from Equation 4 and then a correction term, computed separately, was subtracted from it. The information that the AIBI provided about the stimulus, $I_{\mathrm{AIBI}}$, was computed in a similar way, using the AIBI values in all trials and assuming Gaussian statistics. The information that the PSFP provided about the stimulus, $I_{\mathrm{PSFP}}$, was computed somewhat differently. Because of the Fourier methods involved, PSFP values were drawn from binned distributions, so $I_{\mathrm{PSFP}}$ was computed using the original PSFP bins between 4 and $42 \mathrm{~Hz}$, which correspond to the flutter range; including higher frequencies only increased the uncertainty in frequency and decreased $I_{\mathrm{PSFP}}$. Corrections for finite sampling were also applied in this case (see below). For all information estimates, at least five trials per stimulus value were required.

The significance of all information values was computed through Monte Carlo resampling schemes (Efron, 1982; Press et al., 1992) akin to permutation tests (Siegel and Castellan, 1988). The basic procedure consists of shuffling the order of the trials with respect to the stimulus labels, such that the correspondence between stimulus and response is disrupted, made entirely random, and then recomputing the information values as was done originally, before shuffling. This is done repeatedly, with different shufflings, to obtain the fraction of times that the shuffled information was larger or equal to the original information computed previously from the nonshuffled responses. This fraction gives an estimate of the probability of measuring the original amount of information just by chance, when the actual information is really zero. This is precisely the significance: the probability of measuring the original amount of information when the responses are in fact independent from the stimulus. For all significance estimates, 2000 shufflings were used. In tests using synthetic data generated by a computer, we found that this method to obtain the significance of information was extremely robust: its results were accurate even with small numbers of trials (five) and regardless of the distributions from which the data were drawn. A significant amount of information indicates that a real association between stimulus and response probably exists; the amount of information quantifies the strength of this association.

When information is computed from relatively small numbers of data samples, it is typically biased upward with respect to its true value, especially when binned distributions are used (Treves and Panzeri, 1995; Abbott et al., 1996). All of the information calculations for $I_{\mathrm{R} \text { ATE }}, I_{\mathrm{PSFP}}$, and $I_{\mathrm{AIBI}}$ were extensively cross-validated through computer simulations to minimize such biases. The simulations essentially consisted of three steps: (1) defining mathematical fits or binned distributions that modeled the measured empirical response distributions (for rate, PSFP, or AIBI); (2) generating, from these model distributions, synthetic data sampled exactly like in the experiments, with the same numbers of stimuli and samples; and (3) comparing the information computed from the full model distributions to the information computed from the synthetic sampled data. This comparison revealed how information estimates from sampled data deviate from the true values on average, depending on the numbers of samples and the type and distribution of the responses considered. In practice, this provided two things: first, an error bar for the information, and second, the term to be subtracted from Equation 4, i.e., the bias.

Overall, the average correction for $I_{\mathrm{RATE}}$ and $I_{\mathrm{AIBI}}$ was approximately -0.11 bits, and it was similar for significant and nonsignificant values. The mean correction was larger for $I_{\mathrm{PSFP}}$, because it was computed from binned distributions: on average it was approximately -0.4 bits for significant values and approximately -1.1 bits for nonsignificant ones. As a comparison, the largest nonsignificant $I_{\mathrm{PSFP}}$ in the data presented below (after the correction) was of 0.4 bits. Thus, small, nonsignificant $I_{\mathrm{PSFP}}$ values were the most biased and suffered the greatest corrections. Note also that the uncorrected numbers never exceeded the theoretical maximum equal to $\log _{2}$ of the number of frequencies used. These corrections are important for the results below primarily when $I_{\mathrm{PSFP}}$ is compared with $I_{\mathrm{RATE}}$ or $I_{\mathrm{AIBI}}$; otherwise, they make little difference.

Unless specified otherwise, other statistical comparisons were based on permutation tests (Siegel and Castellan, 1988). Here the underlying idea is practically the same as for the computation of significance described above. Two distributions are thought to differ in some statistic, for instance in their means. To test the significance of the difference, the two distributions are mixed, and two new shuffled distributions, with the same numbers of elements as the original ones, are obtained. Then the difference in the means is recomputed, as done originally. The procedure is repeated many times with different shufflings, and the end result is an estimate of the probability of measuring the original difference in the means just by chance, under the null hypothesis that the two sample distributions actually came from the same source. This powerful procedure may be applied not only to the mean but to other statistics as well. It was used in all pairwise comparisons reported here. For these tests, 5000 permutations were performed; thus $p<0.0002$ was the maximum resolution. Significance was set at the $p<0.01$ level.

\section{RESULTS}

\section{Firing rate and periodicity modulations in S1}

Three monkeys (Macaca mulatta) were trained in the discrimination task. In each trial, the frequencies of two mechanical vibrations delivered successively had to be compared (Romo et al., 1998; Hernández et al., 1997) (Fig. 1; and see Materials and Methods). After training, single neurons were recorded extracellularly while the task was performed (Mountcastle et al., 1990; Romo et al., 1998). In primates, processing of somatosensory information from $\mathrm{S} 1$ to S2 seems to proceed mostly in a serial fashion (Pons et al., 1987, 1992). We recorded in these two areas to assess any differences in the processing or representation of tactile information. In both areas, a neuron was selected for study if, relative to background activity, it responded in any way to the base or comparison stimulus or during the interstimulus interval. For S1 neurons (areas $3 \mathrm{~b}$ and 1) the stimulating probe was placed at the receptive field centers. S2 neurons had large receptive fields, often bilateral, spanning all digits and sometimes even reaching the forearm (Pons et al., 1987, 1992; Sinclair and Burton, 1993; Fitzgerald et al., 1999). Stimuli were always applied at the fingertips and, as illustrated in Figure $1 a$, consisted of trains of short mechanical pulses delivered at various frequencies.

For each neuron, two quantities were computed in each trial: the mean firing rate and the PSFP, which is an estimate of stimulus frequency based on the periodicity of evoked action potentials (see Materials and Methods). We used the PSFP because, just like firing rate, it is a scalar quantity from which stimulus frequency can be estimated on a trial-by-trial basis; however, unlike with firing rate, 
a

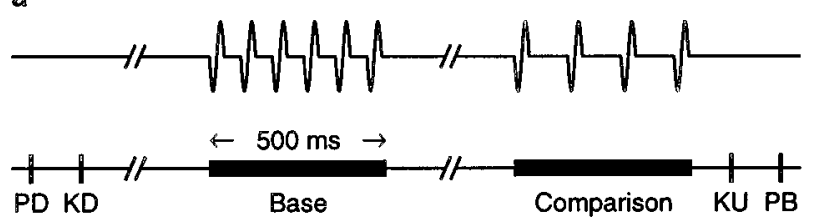

b
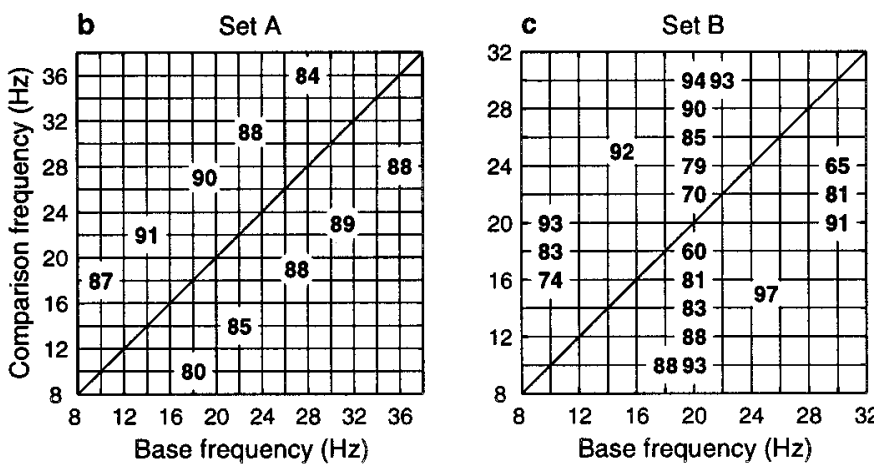

Figure 1. Behavioral paradigm and stimulus sets used. $a$, Schematic diagram of the task. In each trial, the mechanical probe was lowered so that it touched one of the fingertips of the restrained hand ( $P D$, probe down); the monkey reacted, placing its free hand on a lever within 1 sec after indentation $(K D$, key down); after a delay period $(1.5-3 \mathrm{sec})$ the probe oscillated vertically, delivering a series of pulses at a base frequency; after an interstimulus interval $(1-3 \mathrm{sec})$, a second set of pulses was delivered at a comparison frequency; after the end of the comparison stimulus, the monkey had to release the lever within $600 \mathrm{msec}(K U$, key up) and press one of two push-buttons $(P B)$. One button indicated that the comparison frequency was higher than the base, and the other indicated that the comparison was lower than the base. $b, c$, Two stimulus sets frequently used in the experiments. The numbers inside the grid indicate the percentage of correct responses for each base-comparison combination. Set $A$ had constant differences of $8 \mathrm{~Hz}$ between base and comparison. Percentages are based on the performance of three monkeys throughout 350 runs with this set. Set $B$ was designed to vary the difficulty of the task in a more systematic manner. The percentages shown correspond to 42 runs from two monkeys.

the accuracy of this estimation depends on the periodicity of the spike trains. Figure 2, $a$ and $c$, shows examples of S1 spike trains evoked during the base stimulus in individual trials, and Figure 2, $b$ and $d$, shows the corresponding power spectra. The PSFP is simply the center ( $x$ coordinate) of the frequency bin with the most power. As illustrated in these Figures, the PSFP in S1 tends to be the same across trials. This is because the evoked spikes are phase-locked to the individual stimulation pulses. This can be seen more clearly in Figure $2 h$, which shows average $\mathrm{S} 1$ responses triggered at the time of individual pulses, the onset of which occurs at a time lag equal to $0 \mathrm{msec}$. The evoked activity reflects the periodicity of the sensory input. Curves for mean PSFP versus frequency were also obtained; this was done by averaging the PSFP over trials with equal stimulus frequency. These curves are shown in Figure $2 f$. Here the points fall close to the $x=y$ line, confirming that the PSFP typically falls near the stimulus frequency. Curves for mean firing rate versus frequency were also obtained; examples are shown in Figure $2 e$. Notice that these neurons tend to fire more action potentials at higher stimulus frequencies. This was also true for the population: when straight lines were fit (Press et al., 1992) to the rate-versus-frequency data, most neurons had positive slopes, as shown in Figure $2 g$. Variations in mean rate across the tested range of frequencies were similar to those observed previously in somatosensory cortex using paradigms based on other tactile stimuli, such as textured surfaces or tactile motion (Sinclair and Burton, 1991, 1993; Gardner et al., 1992; Romo et al., 1996).

Curves like those of Figure 2, $e$ and $f$, give a rough idea of the strength of association between the stimulus and the evoked variations in firing rate and in PSFP, but comparing them against each other is difficult. Instead, a quantitative measure of association was computed: Shannon's mutual information (Cover and Thomas, 1991; Abbott et al., 1996) (see Materials and Methods). This statistic is useful because it allows a direct comparison between the two kinds of response in the same units, that is, in terms of their capacity to encode stimulus frequency. The maximum amount of information in these experiments was 3 bits.

In S1, the information that the PSFP-i.e., periodic spike timing - provided about stimulus frequency, $I_{\mathrm{PSFP}}$, was extremely high $(1.71 \pm 0.95$ bits, mean $\pm \mathrm{SD} ; 107$ of 129 values were significant, $p<0.01$ ), as can be seen in Figure $2 i$ (right plot). In 12 cases, $I_{\mathrm{PSFP}}$ $>2.8$ bits, which means that by computing the PSFPs of any one of these neurons, on average seven frequencies could in principle be distinguished from each other with $100 \%$ reliability. The spike rasters of S1 neurons seem to provide a faithful representation of the stimulus as it progresses in time (Fig. $2 a, c, h$ ), and the high $I_{\mathrm{PSFP}}$ values agree with this subjective impression. Notice in Figure $2 i$, however, that the mean $I_{\mathrm{PSFP}}$ dropped considerably from area $3 \mathrm{~b}$, which receives the heaviest thalamic projection (Jones, 1975, $1983)$, to area 1 . The average numbers were $1.96 \pm 0.97$ bits for area $3 \mathrm{~b}(n=68)$ and $1.43 \pm 0.86$ bits for area $1(n=61)$, and the difference was highly significant $(p<0.001)$. These $I_{\mathrm{PSFP}}$ values represent upper bounds on the information provided by the PSFP that is available to neurons downstream from $\mathrm{S} 1$, because neuronal mechanisms that may actually implement an approximate Fourier decomposition - for example, operations based on spike train autocorrelations (Cariani and Delgutte, 1996) or intrinsic oscillators (Ahissar and Vaadia, 1990; Ahissar, 1998)_cannot match the accuracy of the numerical methods (Press et al., 1992) used to compute the PSFP.

In contrast to these numbers, the information about stimulus frequency provided by the firing rate, $I_{\mathrm{RATE}}$, was approximately sixfold lower, but certainly not negligible $(0.28 \pm 0.23$ bits; 74 of 129 values were significant). The distribution of values is shown in Figure $2 i$ (left $)$. What order of magnitude for $I_{\mathrm{RATE}}$ should we have expected based on rate curves like those in Figure $2 e$ ? To get a better idea of the correspondence between the rate-versusfrequency curves and $I_{\mathrm{RATE}}$, consider the following idealized but representative example. Suppose the applied stimulus frequency $s$ can take one of eight values, $8,12,16,20,24,28,32$ or $36 \mathrm{~Hz}$, and consider a neuron whose mean firing rate increases linearly with $s$ with a slope of 0.7 spikes, typical of S1 (Fig. $2 g$ ), such that the evoked mean firing rate can be described by:

$$
r(s)=22+0.7 s+\sigma \epsilon .
$$

Here $\epsilon$ represents random Gaussian noise with zero mean and unit variance, so $\sigma$ is the $\mathrm{SD}$ of the mean firing rate. This $\sigma$ is equivalent to the $\sigma$ computed from the experimental data, except that, for simplicity, it is considered independent of frequency $s$. On average, the mean rate of this ideal neuron is $\sim 28$ spikes/sec when $s=8 \mathrm{~Hz}$ and $\sim 47$ spikes/sec when $s=36 \mathrm{~Hz}$; these values are also typical for the minimum and maximum mean rates at which $\mathrm{S} 1$ neurons fired during our experiments. For this idealized typical neuron, when the amplitude of the noise is $\sigma=3.5$ spikes $/ \mathrm{sec}, I_{\mathrm{RATE}}=1 \mathrm{bit}$; when $\sigma=8.7 \mathrm{spikes} / \mathrm{sec}$ (close to the average measured value, as seen in Fig. $5 c$ ), $I_{\mathrm{RATE}}=0.3$ bits; and when $\sigma=16$ spikes $/ \mathrm{sec}$ $I_{\mathrm{RATE}}=0.1$ bits. In comparison, a Poisson process, which provides a reasonable first order model for neuronal firing (Softky and Koch, 1993; Shadlen and Newsome, 1998), would give $I_{\mathrm{RATE}}=0.3$ bits, assuming that it fired at the same mean rates and that spikes were counted in a $500 \mathrm{msec}$ time window. So, for cortical standards, 1 bit corresponds to an extremely reliable neuron, and 0.3 bits should be more or less typical given the experimental parameters and the measured rates. This is in agreement with the information values computed from the data.

Taken together, these results confirm that S1 spikes are precisely time-locked to the stimulation pulses (Mountcastle et al., 1969, 1990; Recanzone et al., 1992), but they also show that although periodic firing can in principle provide a better code for stimulus frequency, firing rate cannot be dismissed. 
Neuron from area 3b

a

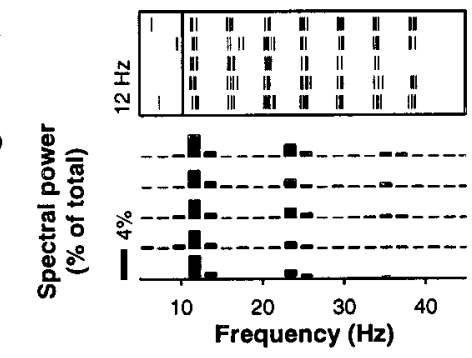

c

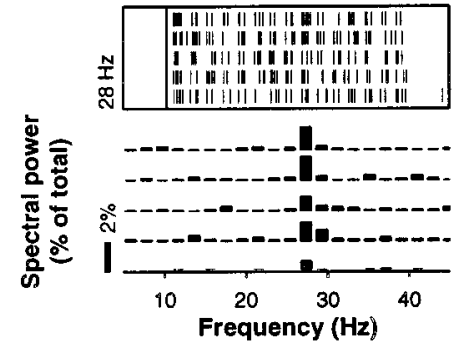

e

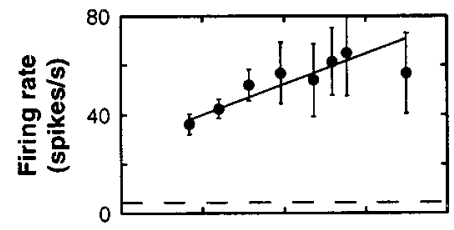

$\mathbf{f}$

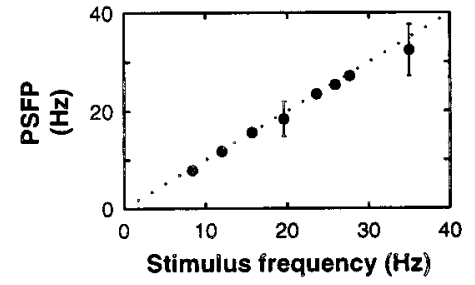

Neuron from area 1
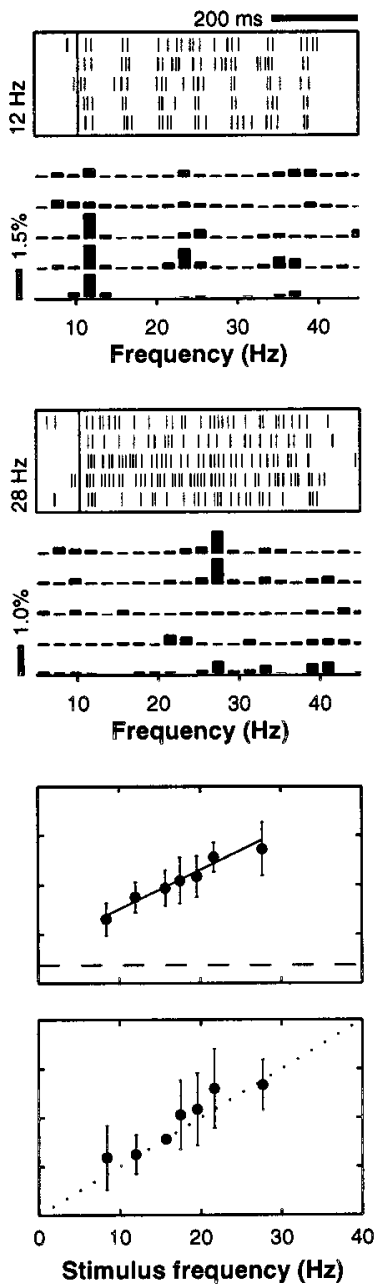

S1 population

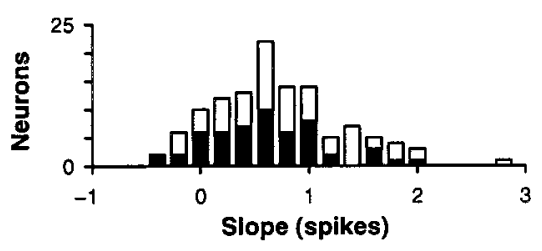

9
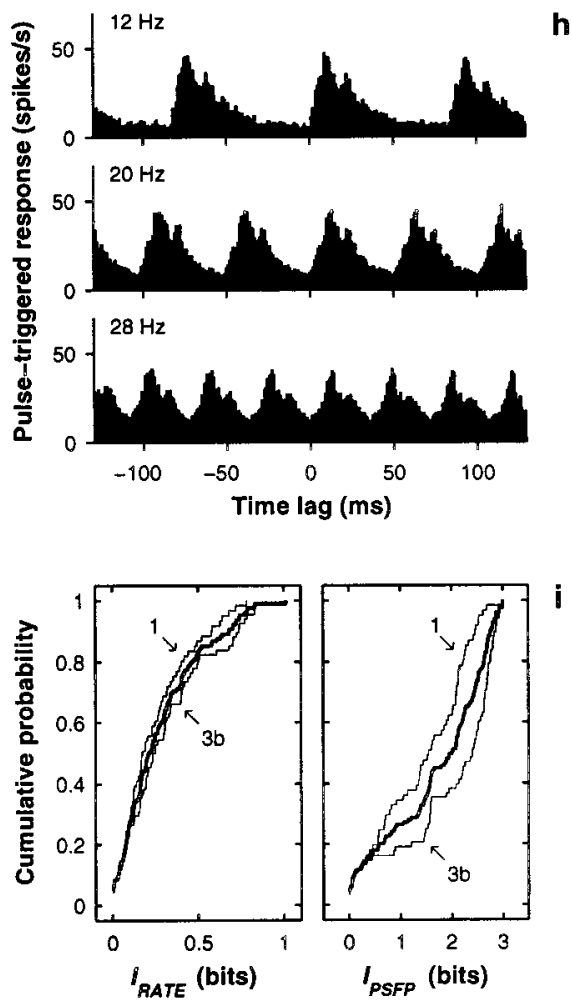

Figure 2. Neuronal responses in S1. Left and middle columns show data, in the same format, from two neurons from areas $3 \mathrm{~b}$ and 1 , respectively. The right column shows population data from 68 neurons in area $3 \mathrm{~b}$ and 61 neurons in area 1 . All plots are based on neuronal activity evoked by the base stimulus. $a$, $c$, Raster plots from five trials in which stimulus frequency was $12 \mathrm{~Hz}(a)$ and $28 \mathrm{~Hz}(c)$. Small vertical ticks indicate spikes; each row corresponds to one trial. The long vertical line indicates stimulation onset. $b, d$, Power spectra of the five spike trains shown immediately above. Power is expressed as percentage of total power across all bins, but only frequencies within the flutter range are shown. $e$, Mean firing rate $( \pm 1$ SD) as a function of stimulus frequency. Continuous line indicates best linear fit; dashed line indicates baseline firing rate, computed in the 800 msec preceding stimulation onset. For the neurons on the left and middle columns, $I_{\mathrm{RATE}}=0.50 \pm 0.10$ and $0.58 \pm 0.09$ bits, respectively (both significant). $f$, Mean PSFP ( \pm 1 SD) as a function of stimulus frequency. The diagonal dotted line indicates equality between $x$ and $y$ axes. For the neurons on the left and middle columns, $I_{\mathrm{PSFP}}=2.71 \pm$ 0.03 and $2.08 \pm 0.04$ bits, respectively (both significant). Because PSFP values were discrete and often distributed bimodally, SDs here suggest more overlap between response distributions than was actually measured. $g$, Distribution of slopes from linear fits to the rate-versus-frequency curves (as in $e$ ). White and black bars correspond to area $3 \mathrm{~b}$ and area 1 neurons, respectively. $h$, Average S1 responses triggered on individual stimulation pulses. The three histograms correspond to stimulation at 12,20 , and $28 \mathrm{~Hz}$ and were constructed from the responses of $89-102 \mathrm{~S} 1$ neurons tested at these frequencies. The $y$ axis indicates the firing rate (in $1 \mathrm{msec}$ time bins), averaged over neurons and trials, $x$ milliseconds before or after the onset of an individual stimulation pulse, where $x$ is called the time lag. Phase-locking is readily apparent at all frequencies. $i$, Cumulative distributions for $I_{\mathrm{R} \text { ATE }}$ and $I_{\mathrm{PSFP}}$. The value on the $y$ axis represents the fraction of neurons with information smaller or equal to the amount indicated on the $x$ axis. Thin lines indicate separate distributions for areas $3 \mathrm{~b}$ and 1 ; thick lines correspond to pooled data sets. Note the different scales on the $x$ axes.

\section{Differences between S1 and S2}

Figure 3 shows results, displayed in the same format as those in Figure 2, for a population of $\mathrm{S} 2$ neurons. The mean strength of rate modulations in $\mathrm{S} 2$ was comparable to that measured in S1: the average $I_{\mathrm{RATE}}$ in $\mathrm{S} 2$ was lower ( $0.14 \pm 0.18$ bits), but the maximum values were still around 1 bit, as shown in Figure $2 i$, and $\sim 20 \%$ of all values (139 of 689) were significant (a calculation similar to the one around Equation 5 in this case gave a typical $I_{\mathrm{RATE}}$ of $\sim 0.2$ bits, assuming Poisson statistics). Thus, considerable rate modulation was also present in S2. However, comparison between S1 and $\mathrm{S} 2$ responses revealed four major differences.

First, a lack of periodicity in S2 was revealed. This can be seen in the spike rasters of Figure 3, $a$ and $c$, and in the pulse-triggered responses of Figure $3 h$. For the latter, the responses of neurons with positive and negative slopes were averaged, which is why the mean firing rates are so similar at the three frequencies shown. Note that phase-locking is hardly noticeable, especially at higher frequencies. Consistent with this, the mean PSFP in this case is practically independent of stimulus frequency, as illustrated in the examples of Figure $3 f$. In quantitative terms, the mean $I_{\mathrm{PSFP}}$ in S2, computed for the spikes evoked during the base stimulus, was an order of magnitude smaller than in S1 ( $0.17 \pm 0.34$ bits $)$, and only 52 of the 689 neurons had values that were significantly different from zero.

Second, S2 contained a larger proportion of neurons that fired most strongly at low stimulation frequencies. The middle column of Figure 3 illustrates such a unit, and $e$ shows that its rate decreases as a function of frequency. As mentioned above, in S1 stronger activity typically occurred at higher frequencies, as shown in Figure $2 e$. When firing rates were fitted (Press et al., 1992) as linear 
Neuron with + slope

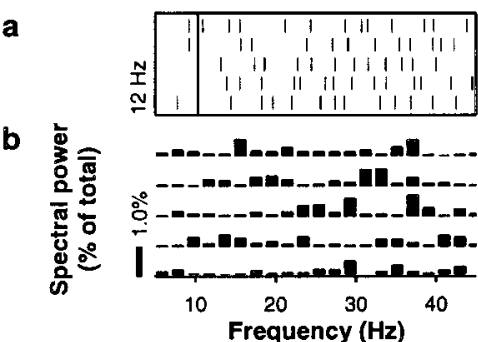

c

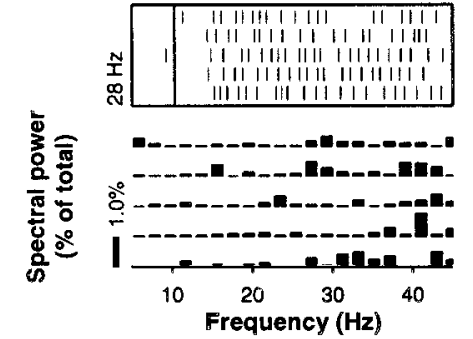

e

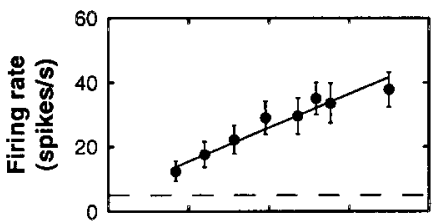

f

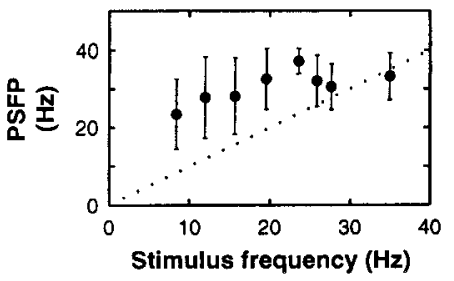

Neuron with - slope
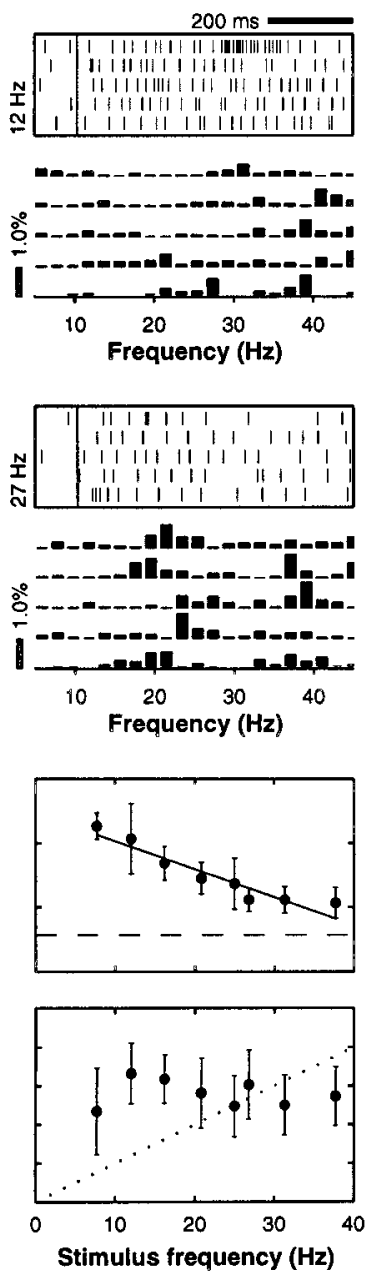

S2 population
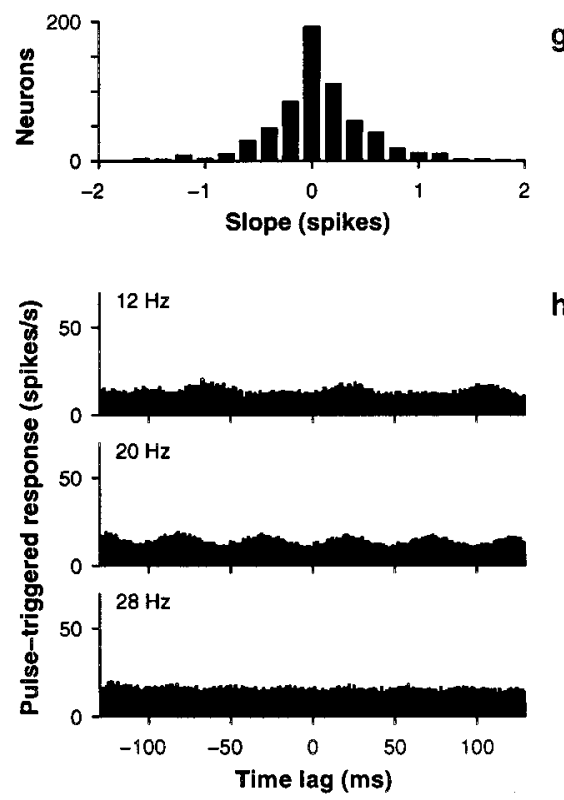

h

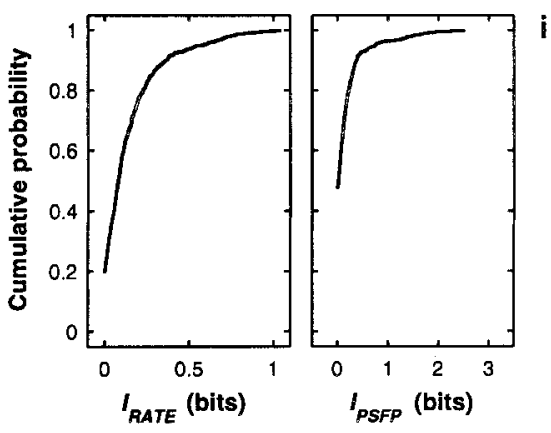

Figure 3. Neuronal responses in S2. Left and middle columns show data from two neurons: the firing rate of one increases with increasing stimulus frequency (positive slope), and the firing rate of the other decreases with increasing stimulus frequency (negative slope). Slopes were extracted from the linear fits shown in $e$. Same format is used as in Figure 2, except in $d$, middle column, frequency was $27 \mathrm{~Hz}$; in $e, I_{\mathrm{RATE}}=0.89 \pm 0.09$ and $0.75 \pm 0.13$ bits for left and middle columns, respectively (both significant); in $h, I_{\mathrm{PSFP}}=0.26 \pm 0.18$ and $I_{\mathrm{PSFP}}=0 \pm 0.30$ bits, for left and middle columns, respectively (both not significant). In $h$, histograms are averages of 250-287 neurons (note same scale as in Fig. 2). Population data in $g$ and $i$ are based on 689 S2 neurons. All data are based on neuronal activity evoked by the base stimulus.

functions of frequency, in S1 only $8 \%(10 / 129)$ of the resulting slopes were negative, whereas in S2 42\% (287/689) of the slopes were negative. This difference can be seen by comparing Figures $2 g$ and $3 g$. Interestingly, a similar transformation between S1 and S2 representations has been reported for textured surfaces (Sinclair and Burton, 1993). Here we should also mention that, in both areas, most response curves were approximately monotonic. First, the linear fits were acceptable $[Q>0.001$; see Press et al., (1992)] in $53 \%(68 / 129)$ and $87 \%(602 / 689)$ of the neurons in S1 and S2, respectively. When Gaussian tuning curves were fitted to the same data, $98 \%$ of the fits were acceptable in both areas (126/129 in S1, $673 / 689$ in S2). This is not surprising, because Gaussian tuning curves had four parameters: baseline, amplitude, center frequency, and width (see Eq. 1). Still, many of the resulting curves were monotonic, because the centers of the best fitting Gaussians were either outside or at the edges of the frequency interval that contained the data. For instance, the area 1 neuron in Figure $2 e$ (right) had a center frequency $C=31 \mathrm{~Hz}$, beyond the highest frequency tested, and a width $\sigma_{\mathrm{G}}=18 \mathrm{~Hz}$. For other neurons, the Gaussian curves fitted better the saturation effects often seen at lowest or highest firing rates. For example, the firing rate of the area $3 \mathrm{~b}$ neuron in Figure $2 e$ (left) is lower for 36 than for $28 \mathrm{~Hz}$, although it has a positive slope. The Gaussian fit for this neuron had center frequency $C=29 \mathrm{~Hz}$ and width $\sigma_{\mathrm{G}}=18 \mathrm{~Hz}$. We considered a neuron as tuned if the limits $C \pm \sigma_{\mathrm{G}}$ were both inside the interval of tested frequencies and if the neuron also had a significant $I_{\mathrm{RATE}}$. The first condition assures that small saturation effects, like that of the area $3 \mathrm{~b}$ neuron in Figure $2 e$ (left), are not counted as actual tuning, and the second one guarantees that the Gaussian curve is significantly different from flat. Few neurons were found that satisfied these criteria: $12 \%(15 / 129)$ in S1 and $1 \%(8 / 689)$ in S2. In conclusion, most S1 and S2 rate-versus-frequency curves were reasonably monotonic, with negative slopes being more common in $\mathrm{S} 2$.

The third difference was that "flat" neurons were more abundant in S2 $(62 \%, 428 / 689)$ than in S1 $(31 \%, 40 / 129)$. Flat neurons had firing rates that did increase or decrease significantly during stimulation, compared with the baseline activity preceding the base stimulus, but were not affected by stimulus frequency, i.e., $I_{\text {RATE }}$ was not significant (here we used $p>0.05$ as a criterion). This was also reflected in the distribution of slopes: a larger fraction of S2 neurons had slopes that were close to zero, as can be seen by comparing Figures $2 g$ and $3 g$. This difference in the proportion of flat neurons could be caused partly by suboptimal stimulation of S2; we have observed that $\mathrm{S} 2$ receptive fields have essentially no preference for one or another fingertip (Fitzgerald et al., 1999), but sometimes they do extend beyond the hand (Pons et al., 1987, 1992; Sinclair and Burton, 1993). 

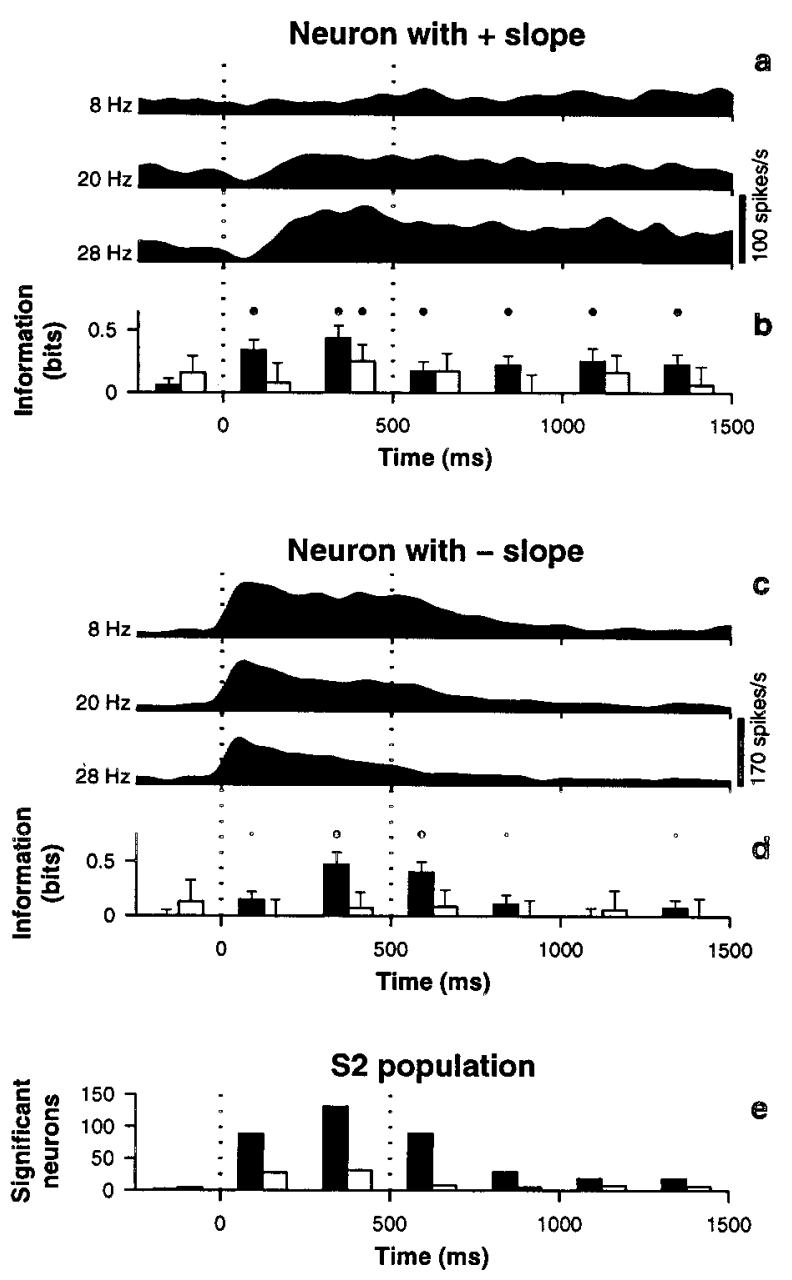

Figure 4. Sustained neuronal responses in S2. The base stimulus turned on at time zero, lasting $500 \mathrm{msec}$; stimulus onset and offset are indicated by dotted vertical lines. Interstimulus interval duration was 1-3 sec. $a$, Spike density histograms of a neuron that fired most strongly at high frequencies (positive slope). For the shown traces, stimulus frequencies were 8,20, and $28 \mathrm{~Hz}$, as indicated. $b$, Information ( $+1 \mathrm{SD})$ carried by the neuron illustrated in $a$ as a function of time. $I_{\mathrm{RATE}}$ (black bars) and $I_{\mathrm{PSFP}}$ (white bars) were computed every $250 \mathrm{msec}$ using the spikes contained in a $250 \mathrm{msec}$ time window centered at the midpoint ( $x$ coordinate) between bars. Large and small dots indicate significance levels of $p<0.01$ and $p<0.05$, respectively. $c$, Spike density histograms of a neuron that fired most strongly at low frequencies (negative slope); same stimulus frequencies as in $a$. $d$, Information carried by the neuron illustrated in $c$ as a function of time. $e$, Number of neurons with significant $(p<0.01)$ information about stimulus frequency as a function of time. Black bars correspond to $I_{\mathrm{RATE}}$ and white bars to $I_{\mathrm{PSFP}}$, as in $b$ and $d$. All spike densities were obtained by convolving the spike trains with a Gaussian kernel of SD equal to $30 \mathrm{msec}$ and averaging over trials of equal frequency.

Fourth, many neurons in S2 either sustained their frequencyspecific responses beyond the base stimulus or displayed them only after stimulus offset, during the interstimulus interval. Figure $4 a$ illustrates this for a neuron with positive slope that maintained significant rate modulation even $1 \mathrm{sec}$ after stimulus offset. Figure $4 c$ shows the activity of another, more typical neuron that had a negative slope and prolonged its response for a few hundred milliseconds. The histograms in Figure $4, b$ and $d$, indicate the amount and significance of $I_{\mathrm{RATE}}$ and $I_{\mathrm{PSFP}}$ for these neurons as a function of time, and the plot in Figure $4 e$ presents the numbers of $\mathrm{S} 2$ neurons with significant information $\left(I_{\mathrm{RATE}}\right.$ and $\left.I_{\mathrm{PSFP}}\right)$ also as a function of time. Notice that $\sim 13 \%(89 / 689)$ of the neurons displayed significant rate modulations in the $250 \mathrm{msec}$ window after stimulus offset. Figure $4 e$ also shows that during this same period, 10 neurons also had significant $I_{\text {PSFP }}$; however, the number expected just by chance was seven. This means that the sustained activity lacked a significant oscillatory component. Significant rate modulations after the stimulus were not observed in S1: four neurons had significant $I_{\text {RATE }}$ during the interstimulus interval, but three were expected just by chance. Therefore, sustained activity was absent in the primary sensory area [compare with Zhou and Fuster (1996, 1997)]. The significance of maintained S2 activity is hard to pinpoint. To perform the task correctly, the monkeys had to store the frequency of the base stimulus in short-term memory (Hernández et al., 1997; Romo et al., 1999). Some prefrontal neurons are also active in this task, throughout or at different points of the interstimulus interval, and their mean firing rates also increase or decrease quasilinearly as functions of stimulus frequency (Romo et al., 1999). Additionally, we found that the sustained modulation in S2 was greatly reduced during passive stimulation, when the stimuli were applied but did not have to be remembered (data not shown). Hence, it is tempting to think that such sustained activity may be related to the working memory requirements of the task, but this is speculative.

\section{A simple compromise between firing rate and timing}

The above results show that, on the basis of single-cell comparisons, firing rate modulations in S2 were somewhat weaker than those in S1 in terms of information content; on average, $I_{\mathrm{RATE}}$ differed by a factor of 2 . However, the difference in terms of periodicity was a factor of 10 . Although in S2 the actual average values of $I_{\mathrm{RATE}}$ and $I_{\mathrm{PSFP}}$ were similar, two points should be stressed: first, that the fraction of neurons with significant $I_{\mathrm{RATE}}$ was twice as high as the fraction of neurons with significant $I_{\mathrm{PSFP}}$, and second, that the $I_{\text {PSFP }}$ values represent upper bounds.

We also checked whether distinctions between frequencies could be made based on the AIBI in each trial. A burst is simply a group of spikes close together in time, like those shown in Figure $2 a$ (left). We defined a burst through a time window $\tau$ such that any two spikes within $\tau$ milliseconds of each other belonged to the same burst. Notice that the rate of bursts and the rate of spikes are correlated-indeed, if $\tau$ is very small each spike equals a burst and the two rates become equal-but grouping by bursts with more than one spike may produce more accurate results than simply counting spikes, especially when long interspike intervals correspond to intervals between consecutive stimulation pulses. The AIBI represents a plausible middle ground between counting the total number of spikes, ignoring their temporal distribution, and taking into account all individual interspike intervals.

For each neuron, the AIBI was obtained in each trial, and the information that the AIBI provided about stimulus frequency, $I_{\text {AIBI }}$, was computed (see Materials and Methods). Parameter $\tau$ was set to optimize the average $I_{\mathrm{AIBI}}$ in S1. It should be borne in mind that, having optimized $\tau, I_{\mathrm{AIBI}}$ is expected to be at least equal to $I_{\mathrm{RATE}}$, because one may always choose $\tau$ close to zero and count each spike as a burst. A positive value of $I_{\mathrm{AIBI}}-I_{\mathrm{RATE}}$ means that additional information is extracted from the timing of spikes, in excess of the information provided by the rate. With an optimal $\tau$ of $20 \mathrm{msec}$, the average $I_{\mathrm{AIBI}}$ in S1 was $0.58 \pm 0.49$ bits $(n=129)$. Thus, although the PSFP was more efficient, the AIBI did capture some of the periodic structure of the spike trains, providing twice as much information as the firing rate alone (McLurkin et al., 1991). This was also true for the maximum values, which were $1.16 \pm 0.09$ bits for $I_{\mathrm{RATE}}$ and $2.29 \pm 0.07$ for $I_{\mathrm{AIBI}}$. In contrast, in $\mathrm{S} 2 I_{\mathrm{AIBI}}$ was indistinguishable from $I_{\mathrm{RATE}}(p>0.49, n=689)$, and the maximum value was $0.65 \pm 0.12$ bits, quite below the maximum $I_{\mathrm{RATE}}$, which was $1.04 \pm 0.07$ bits. Other values of $\tau$ were also tested for S2, but the results were similar: the mean $I_{\mathrm{AIBI}}$ always decreased with increasing $\tau$. Hence, grouping spikes by bursts, which effectively doubled the information about stimulus frequency reported by the firing rate in $\mathrm{S} 1$, was entirely ineffective in S2. This confirms, with a different method, that phase-locking is strong in S1 and extremely weak in S2.

According to these results, neurons immediately downstream from S1 may read out stimulus frequency in at least two ways: either from S1 firing rate modulations or from the periodic struc- 
ture of S1 spike trains. In contrast, for neurons downstream from $\mathrm{S} 2$, the second possibility may not be available. Hence, two areas involved in somatosensory processing could potentially use fundamentally different codes to represent the same quantity. S1 is extremely important for somatosensory processing: lesions in this area cause severe impairments in discrimination and categorization tasks (LaMotte and Mountcastle, 1979; Zainos et al., 1997), and activity driven by direct microinjection of electrical current into S1 may trigger sensory percepts that probably resemble natural sensations quite closely (Romo et al., 1998; Wickersham and Groh, 1998). Therefore, the crucial question is whether neurons downstream from S1 read out its periodicity and are affected by it. We performed other experiments to try to address this issue.

\section{Context-dependent modulations of activity}

In general, the attentional state of a subject performing a task may have a strong influence on the neurons involved in it; neuronal responses are often enhanced when attention is focused on a sensory feature that the neurons react to (Hsiao et al., 1993; McAdams and Maunsell, 1999; Treue and Martínez-Trujillo, 1999). We wondered whether spike periodicity or firing rate would be subject to similar modulatory effects. The same sets of stimuli used for discrimination - the active condition-were also delivered passively to the monkeys. During passive stimulation the responding arm was restrained, no behavioral reaction was required, and no reward was delivered.

Figure 5 compares S1 activity evoked during the comparison stimulus in active and passive conditions. Figure $5 a$ shows that the mean $I_{\mathrm{R} \text { ATE }}$ was significantly higher in the active condition $(0.42 \pm$ 0.35 bits in active, $0.27 \pm 0.23$ bits in passive; $n=50$ neurons with significant information in at least one of the conditions; $p<$ 0.0004); indeed, most points fall above the equality line. Other measures of neuronal activity also showed significant variations across conditions. Figure $5 c$ shows the average variability in firing rate across trials, $\langle\sigma\rangle$, in the two conditions. In this case most points fall below the diagonal line, indicating that variability in firing rate was significantly smaller during active discrimination $(\langle\sigma\rangle$ was $8.9 \pm$ $4.2 \mathrm{spikes} / \mathrm{sec}$ in active, vs $10.5 \pm 4.6 \mathrm{spikes} / \mathrm{sec}$ in passive; $n=77$ neurons tested in the two conditions; $p<0.0002)$. Across conditions, changes in the signal-to-noise ratio (Eq. 2), which is a simple function of the firing rates, were strongly correlated with changes in $I_{\mathrm{RATE}}$ (linear correlation coefficient was $0.98, p<0.0002$ ). Thus, with all the measures tested we arrived at the same conclusion: the firing rate in $\mathrm{S} 1$ is a more reliable signal during discrimination than during passive stimulation.

We were concerned about this result, however, because we had not taken into account the correlations among neurons, i.e., the stimulus-independent co-fluctuations in numbers of spikes fired. For certain changes in the correlations, the information about stimulus frequency transmitted jointly by the rates of multiple neurons might have actually decreased, despite an increase in the information conveyed by individual neurons (Shadlen and Newsome, 1998; Zohary et al., 1994; Abbott and Dayan, 1999). Two additional results indicated that this was not the case. First, we measured $\rho$, the linear correlation coefficient between pairs of simultaneously recorded neurons averaged over all pairs. For each pair, the coefficient was calculated using Equation 3, and a mean over all pairs was computed. We found that $\rho$ was actually smaller in the active condition, although the difference was not significant $(0.10 \pm 0.18$ in active, $0.16 \pm 0.21$ in passive; $n=84$ pairs tested in $\mathrm{S} 1 ; p>0.037)$. Second, we also computed the information provided jointly by the firing rates of pairs of neurons recorded simultaneously, which takes into account their pairwise correlation, and again we observed, on average, a significant increase in information about stimulus frequency in the active condition with respect to the passive $(p<0.0002)$.

Very similar differences between rate modulation in active and passive conditions were obtained in S2. Figure $6 a$ and $c$, illustrates this for $I_{\mathrm{RATE}}$ and $\langle\sigma\rangle$, but the same was also true for the signalto-noise ratio and other measures of activity (Fig. 6, see legend).
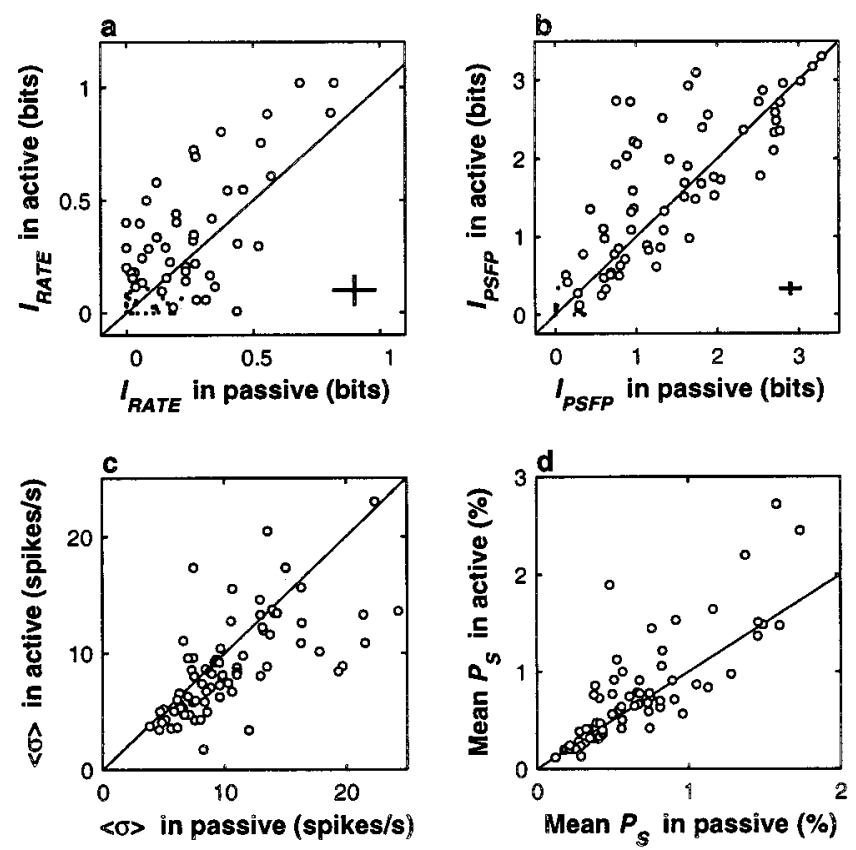

Figure 5. Behavioral context modulates neuronal activity in S1. In all plots, responses during active discrimination ( $y$ axes) are compared with responses during passive stimulation ( $x$ axes). Diagonal lines indicate equality between $x$ and $y$ axes. All comparisons are based on the responses of 77 S1 neurons tested in both situations. All quantities were computed from the responses to the comparison stimulus. $a$, The mean $I_{\mathrm{RATE}}$ was significantly higher during active discrimination $(p<0.0004)$; note that higher values tend to fall above the $x=y$ line. Circles correspond to 50 neurons with $I_{\mathrm{RATE}}$ significantly different from zero in at least one of the two conditions, and dots indicate nonsignificant neurons. Crosses indicate the mean uncertainty in the information values; they correspond to \pm 1 average SD in each direction. $b$, Circles correspond to 63 neurons with $I_{\mathrm{PSFP}}$ significantly different from zero in at least one of the two conditions, and dots indicate nonsignificant neurons. The mean $I_{\text {PSFP }}$ was higher during active discrimination, and the effect was close to but below the significance threshold $(p>$ $0.025)$. Crosses correspond to \pm 1 average SD in each direction. $c$, Trial-totrial variability in the firing rate, quantified by $\langle\sigma\rangle$, was significantly smaller during active discrimination $(p<0.0002)$; note that small values, which correspond to higher reliability, tend to fall below the $x=y$ line. $d$, The mean amplitude of the Fourier spectrum at the stimulus frequency (mean $\left.P_{\mathrm{S}}\right)$ was significantly higher during active discrimination $(p<0.005)$. This indicates that in this condition, the evoked spikes were more phase-locked to the stimulus.

Interestingly, the sustained responses after the offset of the base stimulus exhibited similar but larger effects (data not shown). Regarding the correlation coefficients in S2, again, no difference was found between active and passive conditions ( $\rho$ was $0.07 \pm 0.20$ in active and $0.08 \pm 0.21$ in passive; $n=126$ pairs tested in $\mathrm{S} 2 ; p>$ 0.7 ), and the information carried jointly by the firing rates of pairs of neurons was also significantly higher during active discrimination $(p<0.0002)$. Therefore, the behavioral context of the task definitely had an impact on the evoked firing rates of S1 and S2 neurons: the numbers of spikes produced were significantly more regular across trials during active discrimination.

The periodicity of evoked spikes in S1 was also different in active and passive tests, although the changes seemed more subtle than for rate. This is shown in Figure $5 b$. Here a disproportionate number of data points seem to fall above the equality line, in agreement with the finding that the mean $I_{\mathrm{PSFP}}$ was larger in the active condition $(1.62 \pm 0.90$ in active, $1.45 \pm 0.85$ in passive; $n=$ $63 \mathrm{~S} 1$ neurons with significant $I_{\mathrm{PSFP}}$ in at least one condition), but the effect did not reach the significance criterion of $0.01(p>$ $0.025)$. However, we also compared the mean power at stimulus frequency (mean $P_{\mathrm{S}}$ ) across conditions. This quantity is just the percentage of power at the frequency bin that includes the stimulus frequency, averaged over all trials (see Materials and Methods). The data are shown in Figure $5 d$. The mean $P_{\mathrm{S}}$ was also larger 

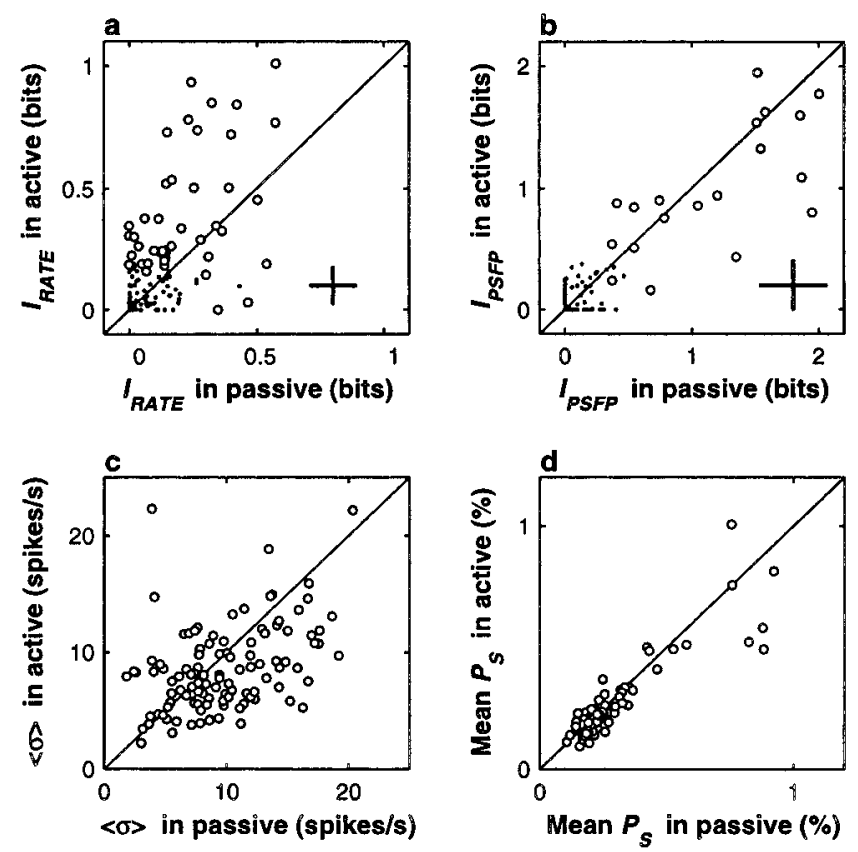

Figure 6. Behavioral context modulates neuronal activity in S2. In all plots, responses during active discrimination ( $y$ axes) are compared with responses during passive stimulation ( $x$ axes). Diagonal lines indicate equality between $x$ and $y$ axes. All comparisons are based on the responses of 108 S2 neurons tested in both situations. Format is the same as in Figure 5, except that all quantities were computed from the responses to the base stimulus. $a$, The mean $I_{\mathrm{R}}$ was significantly higher during active discrimination $(p<0.0002)$; note that higher values tend to fall above the $x=y$ line. Circles correspond to 43 neurons with $I_{\mathrm{R} \text { ATE }}$ significantly different from zero in at least one of the two conditions, and dots indicate nonsignificant neurons. Crosses indicate the mean uncertainty in the information values; they correspond to \pm 1 average SD in each direction. $b$, Circles correspond to 19 neurons with $I_{\mathrm{PSFP}}$ significantly different from zero in at least one of the two conditions, and dots indicate nonsignificant neurons. The mean $I_{\mathrm{PSFP}}$ was not significantly different in the two conditions $(p>0.11)$. Crosses correspond to \pm 1 average $\mathrm{SD}$ in each direction. $c$, Trial-to-trial variability in the firing rate, quantified by $\langle\sigma\rangle$, was significantly smaller during active discrimination $(p<0.0062)$; note that small values, which correspond to higher reliability, tend to fall below the $x=y$ line. $d$, The mean amplitude of the Fourier spectrum at the stimulus frequency (mean $\left.P_{\mathrm{S}}\right)$ did not change across conditions $(p>0.06)$.

during active discrimination $(0.72 \pm 0.53 \%$ in active, $0.62 \pm 0.38 \%$ in passive; $n=77 \mathrm{~S} 1$ neurons), and in this case the effect was significant $(p<0.005)$. Thus, the timing of evoked S1 spikes relative to the stimulation pulses was more regular during active discrimination; tighter phase-locking occurred in this condition.

Figure $6, b$ and $d$, shows that in contrast to S1, no changes in periodicity were detected in S2 in terms of $I_{\mathrm{PSFP}}$ and mean $P_{\mathrm{S}}$ (Fig. 6 , see legend). The same happened for the mean power at the PSFP and for the mean power at twice the stimulus frequency.

Runs of passive tests were applied in blocks, typically after a block of active discrimination trials. Thus, we considered the possibility that the results of this section might have been corrupted by some sort of systematic drift in the recordings, such that later tests tended to be, for instance, more noisy. However, each neuron was typically tested in more than two conditions, so we were able to run the same battery of statistical comparisons on experiments of the same type, active or passive, testing for differences between early and late experimental runs. For example, if three runs (complete blocks of trials) were collected with the sequence active, passive, active, statistical tests were performed between the passive run and the first active run, and the same tests were repeated for the second and first active runs, as a control. Across the neural population, no significant effects were obtained in any of the control comparisons, showing that the described differences between active and passive conditions were not caused by drift artifacts.

In summary, these experiments showed an attentional or a con- textual enhancement of neural activity. In both S1 and S2, the firing rate encoded stimulus frequency better when the stimulus guided the animal's behavior, in the sense that rate provided more information about the relevant stimulus feature. The periodicity of the evoked spikes did not change with behavioral context in S2, but it did so in S1. This was surprising and indicates that spike timing may be influenced by attention or behavioral context (Steinmetz et al., 2000). However, at the level of S1, these results do not favor one neural code over the other.

\section{Responses to aperiodic stimuli}

Two of the monkeys also discriminated the average frequencies of aperiodic stimuli (Romo et al., 1998) (see Materials and Methods). In this situation, the same numbers of pulses corresponding to each stimulus frequency were delivered in the $500 \mathrm{msec}$ stimulation period, but the times between pulses were random and varied from trial to trial. To obtain a reward, the monkeys had to compare correctly the average frequencies of the base and comparison stimuli, just as with periodic vibrations. These animals did not go through a retraining period; they were able to perform the task from the initial runs. Because S1 neurons emit spikes that are reliably phase-locked to individual stimulation pulses, aperiodic stimuli impose a timing between phase-locked spikes or bursts of spikes that, by design, varies randomly within the stimulation period and across trials. Similar random timing can also be imposed directly through intracortical microstimulation (Romo et al., 1998).

The monkeys' performance in this task only decreased slightly compared with discrimination of periodic stimuli: overall, 88 versus $80 \%$ correct (Romo et al., 1998). We investigated whether neuronal responses paralleled this similarity. Figure 7, $a$ and $b$, shows the responses of an S1 neuron to periodic and aperiodic stimuli at two frequencies. This neuron responded quite faithfully to individual stimulation pulses. Notice the regular interspike intervals in the periodic condition, in Figure $7 a$, and the much more variable spike trains elicited in the aperiodic condition, in Figure $7 b$. Figure $7 c$ shows that for any given neuron, $I_{\mathrm{RATE}}$ could vary somewhat from the periodic to the aperiodic situation, but on average, firing rate modulations in S1 were indistinguishable across conditions $\left(I_{\mathrm{RATE}}=0.44 \pm 0.28\right.$ bits for periodic, $0.38 \pm 0.25$ bits for aperiodic; $n=31 \mathrm{~S} 1$ neurons tested in both conditions and with significant $I_{\mathrm{RATE}}$ in at least one of them; $\left.p>0.19\right)$. Differences were slightly larger in $\mathrm{S} 2\left(I_{\mathrm{RATE}}=0.37 \pm 0.22\right.$ bits for periodic, $0.22 \pm 0.17$ bits for aperiodic; $n=13 ; p>0.055)$, but fewer samples were available. These results show that in the two areas, firing rate was, on average, similarly modulated by frequency in periodic and aperiodic conditions.

Not surprisingly, in these experiments $I_{\mathrm{PSFP}}$ practically vanished: of $41 \mathrm{~S} 1 / \mathrm{S} 2$ neurons with significant $I_{\mathrm{PSFP}}$ in the periodic condition, only one had a significant value in the aperiodic condition. The same thing happened with the mean power at the PSFP, at the mean stimulus frequency and at twice the mean stimulus frequency. This was expected and simply showed that no consistent modulations in periodicity are seen with aperiodic stimulation; the Fourier spectrum shows no regularity from one trial to the next.

What about bursts of spikes; could they provide a reliable measure of mean stimulus frequency for aperiodic stimuli? In the periodic condition, the AIBI of S1 neurons carried more information than the rate, as has been described. The AIBI of S1 neurons also provided significant information in the aperiodic condition but, as shown in Figure $7 d, I_{\text {AIBI }}$ in this case was significantly smaller than with periodic stimulation $(0.71 \pm 0.47$ in periodic, $0.32 \pm 0.18$ in aperiodic; $n=31 \mathrm{~S} 1$ neurons tested in both conditions and with significant $I_{\mathrm{AIBI}}$ in at least one of them; $p<$ 0.0002); most data points fall below the equality line. The key observation here is that with aperiodic stimuli, $I_{\mathrm{AIBI}}$ was, on average, slightly smaller than $I_{\mathrm{RATE}}$, and this was the case whether all neurons or only those with significant information were compared. This can be appreciated by comparing the $y$-axis values in Figure 7, $c$ and $d$. Comparisons using bursts of other sizes were also made-we used $\tau=20,15,10$, and $5 \mathrm{msec}$ - but the results were 


\section{Single S1 neuron}
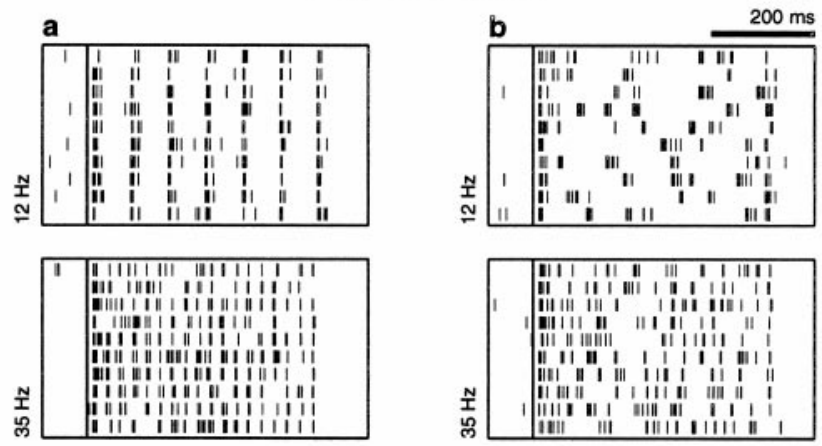

\section{S1/S2 population}
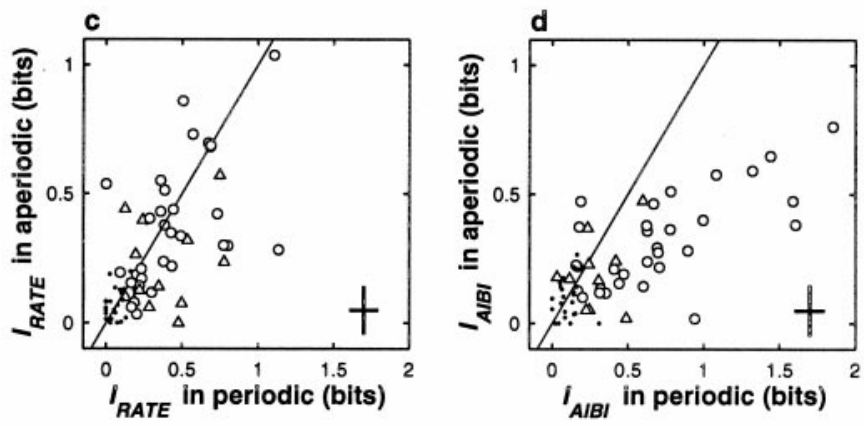

Figure 7. Neuronal responses to periodic and aperiodic stimuli. The four raster plots at the top show spike trains from an S1 neuron that was tested with periodic $(a)$ and aperiodic $(b)$ stimuli at frequencies of 12 and $35 \mathrm{~Hz}$, as indicated. Each set of responses includes 10 trials collected during active discrimination. For a given stimulus frequency, the train of stimulation pulses was identical for all periodic trials but was different for all aperiodic trials. However, at a given mean frequency, the total number of pulses delivered was the same in both conditions. Long vertical lines indicate stimulus onset. In the periodic condition the neuron had $I_{\mathrm{RATE}}=0.67 \pm$ 0.10 and $I_{\mathrm{AIBI}}=1.44 \pm 0.10$ bits; with aperiodic stimulation $I_{\mathrm{RATE}}=$ $0.70 \pm 0.10$ and $I_{\mathrm{AIBI}}=0.65 \pm 0.11$ bits. $I_{\mathrm{RATE}}(c)$ and $I_{\mathrm{AIBI}}(d)$ were computed for $41 \mathrm{~S} 1$ and $30 \mathrm{~S} 2$ neurons tested with periodic and aperiodic stimuli. In both panels, circles and triangles correspond to S1 and S2 neurons, respectively, with significant information in at least one of the two conditions (periodic or aperiodic), and small dots indicate S1 and S2 neurons that had nonsignificant values in the two conditions. Diagonal lines indicate equal values in the two axes. Crosses on the bottom right corners indicate \pm 1 average $\mathrm{SD}$ in each direction. $I_{\mathrm{RATE}}$ did not change across conditions, in either area; data points in $c$ are distributed symmetrically around the diagonal line. $I_{\text {AIBI }}$ was significantly larger with periodic stimulation; data points in $d$ tend to fall below the diagonal. With aperiodic pulses, $I_{\mathrm{AIBI}}$ was similar to $I_{\mathrm{RATE}}$; the $y$-axis values in $c$ and $d$ are similar (see Results).

the same: collecting bursts rather than single spikes provided no additional information about stimulus frequency. Optimizing individually the $\tau$ of each neuron did not increase the information significantly either. Hence, for aperiodic stimuli, clustering the spikes into bursts was just as efficient as ignoring the interspike intervals altogether. For the few S2 neurons tested, there was no significant difference in the mean $I_{\mathrm{AIBI}}$ values across conditions (periodic versus aperiodic), and these values were similar to those of $I_{\text {RATE }}$ (Fig. $7 c, d$, triangles).

Discrimination of periodic and aperiodic stimuli corresponds to slightly different tasks; in fact, the two types of stimuli can be distinguished easily by human subjects. This means that at least some information about the temporal structure of the stimuli is readily accessible perceptually. Therefore, these results cannot exclude the possibility that temporal information is used to construct the neural representation of stimulus frequency, even in the aperiodic condition. However, they provide two conclusions: first, that constructing a neural representation of stimulus frequency based on the temporal patterns of spikes evoked during aperiodic stimulation would require read-out mechanisms more powerful than simply identifying bursts of spikes in single cells, and second, that firing rate could provide a neural code for frequency common to the two tasks and the two areas.

\section{Covariations between neuronal and behavioral responses}

As mentioned in the introductory remarks, earlier studies pointed out a close match between the discriminability of flutter frequencies and the observed variance in the phase at which spikes are evoked by periodic stimuli (Mountcastle et al., 1969; Recanzone et al., 1992). This relationship was only a theoretical possibility, because the neurophysiological and psychophysical data that were compared had been collected in different experiments. However, a similar but direct comparison was possible using our data, because they were collected from behaving animals whose psychophysical performance was being monitored. If the periodicity of S1 spikes is important for frequency discrimination, then a subject should indeed be more likely to discriminate correctly when S1 neurons happen to fire spike trains with a highly periodic structure. This is the crux of the following analysis. We compared neuronal and behavioral responses on a trial-by-trial basis to try to detect any covariations between them. The results below apply to responses computed from neuronal activity evoked during the comparison stimulus. For the base stimulus, no significant effects were found for any quantity, which is not surprising considering the short-term memory component of the task.

The main idea was to compare neuronal responses during correct discriminations (hits) with responses during error trials. Because errors were much less frequent than hits, responses obtained for different conditions, i.e., for different combinations of base and comparison stimulus frequencies, had to be standardized and pooled, as described in Materials and Methods. In all data collection runs, two types of trials were considered separately. In trials of type 1 , the frequency of the comparison stimulus was lower than the frequency of the base, and in trials of type 2, the frequency of the comparison was higher than the frequency of the base. Thus, for each run, two comparisons were made, one for each set of trials of the same type. In both cases the mean standardized response in error trials was compared with the mean standardized response in hit trials, and the significance of the difference was determined. Figure 8, $a$ and $c$, illustrates this procedure for a single S1 neuron, and Figure 9, $a$ and $c$, illustrates it for a single $\mathrm{S} 2$ neuron. Here $H$ and $E$ indicate hit and error categories, respectively, and the subscript indicates the type of trial. Each dot corresponds to a single trial, and the horizontal bars indicate the means for hits and errors in the corresponding categories. In both Figures, the difference between panels $a$ and $c$ lies in the quantity considered as the response.

To detect systematic variations in periodic spike timing, we computed standardized versions of the PSFP amplitude, of $P_{\mathrm{S}}$, and of the amplitude of the power spectrum at twice the stimulus frequency. These three quantities tend to increase the closer a spike train is to a perfectly periodic arrangement, so high values should correspond to better likelihood for correct discrimination, if periodicity is related to performance. In most S1 and S2 cells, these quantities were the same in hit and error trials, as illustrated in Figures $8 c$ and $9 c$. We did find four S1 neurons for which the mean standardized $P_{\mathrm{S}}$ was significantly different for hit and error trials, but this number of neurons was not significantly different $(p>0.21)$ from that expected by chance under the null hypothesis that hit and error responses come from the same distribution (among 238 S1 neurons, 2.38 significant tests at the 0.01 level were expected by chance). In other words, the result was not significant. The same was true for the three measures of periodicity, in both S1 and S2, and for type 1 and type 2 trials. The periodicity of spikes in single neurons showed no detectable covariations with behavior.

This negative result, however, was obtained by testing the neurons one at a time, but if higher periodicity tends to produce better performance, then across the population, standardized responses might show a tendency to be larger in hit trials than in error trials. 
Single S1 neuron

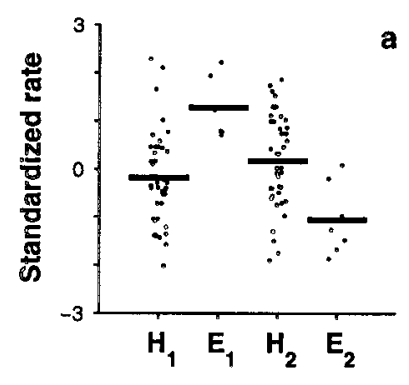

$\mathbf{a}$
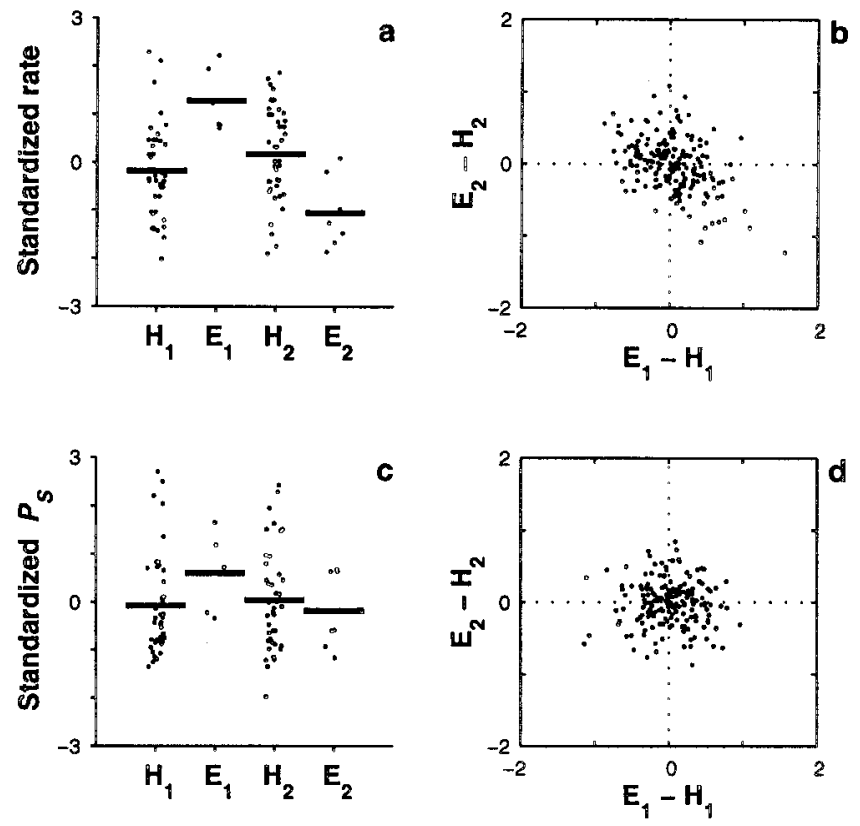

Figure 8. Single-trial covariations between behavioral responses and neuronal responses in S1. This analysis was based on the neuronal activity evoked by the comparison stimulus. $a$, Standardized firing rates (dots) for a single S1 neuron are shown subdivided into four categories: hits $\left(H_{1}\right)$ and errors $\left(E_{1}\right)$ when the frequency of the base stimulus was higher than the frequency of the comparison stimulus (type 1 trials), and hits $\left(H_{2}\right)$ and errors $\left(E_{2}\right)$ when the frequency of the comparison stimulus was higher than the frequency of the base (type 2 trials). Thick horizontal lines represent mean values for each category. For this neuron, the mean values in error trials were significantly different $(p<0.002)$ from the mean values in hit trials of the same type. This neuron had a positive slope of $1.17 \pm 0.12$ spikes. $b$, The standardized firing rate reveals a negative correlation between error types in S1. Each point represents one of 191 S1 neurons with at least five errors of each type. For each neuron, the mean difference in standardized rate between errors and hits for type 2 trials $\left(E_{2}-H_{2}\right)$ was computed and plotted versus the mean difference for type 1 trials $\left(E_{1}-\right.$ $\left.H_{1}\right)$. The population shows a negative correlation between error types: Pearson's linear correlation coefficient was $-0.42(p<0.0002)$. Dotted lines indicate the origin. $c$, Standardized power at the stimulus frequency $\left(P_{\mathrm{S}}\right)$ for the neuron illustrated in $a$, with trials subdivided into the same four hit/error categories. The differences in mean between hits and errors of the same type were not significant, although this neuron had significant $I_{\mathrm{PSFP}}$ $(0.80 \pm 0.14$ bits $) . d$, The standardized $P_{\mathrm{S}}$ shows no correlation between error types in $\mathrm{S} 1$. The plot shows mean differences in standardized $P_{\mathrm{S}}$ between errors and hits for type 2 versus type 1 trials for $184 \mathrm{~S} 1$ neurons. The correlation coefficient was practically zero $(0.008, p>0.9)$. Dotted lines indicate the origin.

Nevertheless, no such trend was observed. This is illustrated in Figures $8 d$ and $9 d$. In these plots, each point corresponds to one neuron. Each $x$ coordinate is the difference between the mean of all type 1 error trials and the mean of all type 1 hit trials, using the standardized $P_{\mathrm{S}}$ as a response, and the $y$ coordinate is the same quantity but for type 2 trials. Observe that the clouds of points are symmetric and centered at 0 in both directions. This is because, on average, differences between responses in hits and errors were not significantly different from zero and were not correlated across types of trials either: for the S1 population in Figure $8 d$, the correlation coefficient was practically zero $(0.008, p<0.9)$, and for the S2 population in Figure $9 d$, the correlation coefficient was -0.23 , but it was not significantly different from zero $(p>0.025)$. Similar results were obtained when these tests were repeated using the standardized power at the PSFP or the standardized power at twice the stimulus frequency. No significant covariations between periodicity and behavior could be detected in either area.

In contrast, the numbers of evoked spikes did show significant covariations with behavioral performance. Figure $8 a$ shows data from an S1 neuron with large differences between the means of the
Single S2 neuron
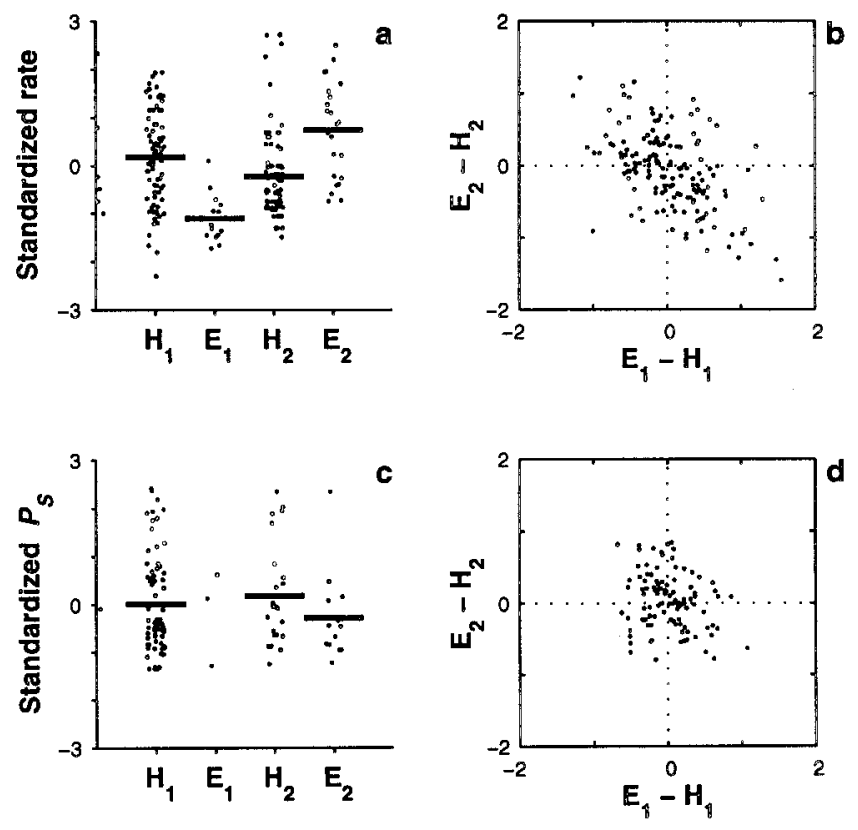

Figure 9. Single-trial covariations between behavioral responses and neuronal responses in $\mathrm{S} 2$, based on the neuronal activity evoked by the comparison stimulus. Same format as in Figure 8. $a$, Standardized firing rates (dots) for a single S2 neuron are shown subdivided into four categories: hits $\left(H_{1}\right)$ and errors $\left(E_{1}\right)$ when the frequency of the base stimulus was higher than the frequency of the comparison stimulus (type 1 trials), and hits $\left(\mathrm{H}_{2}\right)$ and errors $\left(E_{2}\right)$ when the frequency of the comparison stimulus was higher than the frequency of the base (type 2 trials). Thick horizontal lines represent mean values for each category. For this neuron, the mean values in error trials were significantly different $(p<0.0002)$ from the mean values in hit trials of the same type. $b$, The standardized firing rate reveals a negative correlation between error types in S2. Each point represents one of $128 \mathrm{~S} 2$ neurons with at least five errors of each type. For each neuron, the mean difference in standardized rate between errors and hits for type 2 trials $\left(E_{2}-H_{2}\right)$ was computed and plotted versus the mean difference for type 1 trials $\left(E_{1}-H_{1}\right)$. The population shows a negative correlation between error types: Pearson's linear correlation coefficient was -0.52 ( $p<$ $0.0002)$. Dotted lines indicate the origin. $c$, Standardized power at the stimulus frequency $\left(P_{\mathrm{S}}\right)$ for the neuron illustrated in $a$, with trials subdivided into the same four hit/error categories. The differences in mean between hits and errors of the same type were not significant. $d$, Mean differences in standardized $P_{S}$ between errors and hits for type 2 versus type 1 trials for 103 S2 neurons. There was a small, nonsignificant correlation of $-0.23(p>0.025)$. Dotted lines indicate the origin.

$H$ and $E$ categories. This neuron had a positive slope of $1.17 \pm 0.12$ spikes. The significant difference between $H_{1}$ and $E_{1}$ means that at any given comparison frequency lower than the base, on average, the chances of observing an error were higher when the neuron fired more spikes than usual for the given comparison frequency. This association was not a rare event. Among 231 runs that had at least five type 2 errors, we found 11 S1 neurons whose average standardized rates were significantly different in hit and error trials. This number may appear small, but with 231 samples the chances of finding at least 11 significant values when no real difference exists between two conditions is $<3$ in $10^{5}$ (binomial distribution with $p=0.01$ ). Among the 219 runs with sufficient type 1 errors, only four neurons with significant differences were found, which was within the range expected by chance $(p>0.18)$, but there was additional evidence for the firing rate being related to behavior. In this case, a significant effect was observed across the population: the differences between standardized responses for hits and errors were significantly anticorrelated across trial types. This is shown in Figure $8 b$. In this plot the cloud of points is not symmetric; its correlation coefficient is $-0.42(n=191, p<0.0002)$. When a neuron fired, for instance, more spikes in incorrect versus correct discriminations in type 1 trials, it typically fired fewer spikes in incorrect versus correct discriminations of the opposite type. This 
result and the 11 neurons with significant differences in type 2 trials demonstrate a link between the firing rate of S1 neurons and psychophysical behavior.

In $\mathrm{S} 2$ the relationship between rate and behavior was even more evident. In type 1 trials, 28 of 321 neurons had significant differences between hits and errors, and in type 2 trials the numbers were 16 out of 206. The probability of finding these many by chance was, in both cases, $<10^{-9}$. As can be seen in Figure $9 b$, differences across trial types were negatively correlated in this area too. These anticorrelation patterns would be expected if a comparison between the average rates of two neuronal populations underlaid the comparison between frequencies required by the discrimination process (Salinas and Romo, 1998), but this does not exclude other possibilities.

\section{DISCUSSION}

In these experiments, we examined the responses of S1 and S2 neurons to mechanical vibrations applied to the fingertips. Our aim was to determine, in our laboratory task where the only relevant stimulus feature is temporal frequency, what attributes of the evoked neuronal activity are important for behavior. In our simplified task this amounts to finding out what is the neural code for stimulus frequency. We specifically examined the hypothesis that such a code is constructed by some neural mechanism that reads out the periodic interspike intervals of the spike trains evoked in S1. As discovered in earlier work, the periodicity of spikes evoked by flutter vibrations was extremely prominent and reliable in this area. Unfortunately, however, we could not determine with certainty whether their precise timing plays a significant functional role in frequency discrimination. Previous studies contemplated a code for flutter frequency based exclusively on periodicity (Mountcastle et al., 1967, 1969, 1990; Talbot et al., 1968; Recanzone et al., 1992). What we did find, instead, is evidence that firing rate plays a significant role in encoding flutter frequency.

The evidence is as follows. First, rate modulations were widespread. We found that, contrary to earlier reports (Mountcastle et al., 1990; Recanzone et al., 1992), the firing rate of neurons in primary areas $3 \mathrm{~b}$ and 1 varied significantly as a function of flutter frequency (Fig. 2e,i). S2 neurons showed roughly similar rate modulations in terms of the information that rate provides about stimulus frequency (Fig. 3e,i), but interestingly, neurons firing most intensely at low frequencies were much more common in S2 than in $\mathrm{S} 1$ (compare Figs. $2 g$ and $3 g$ ). In some $\mathrm{S} 2$ neurons, rate modulations also persisted beyond the end of the stimulus (Fig. 4). The information conveyed by the rate was significant in $\sim 57$ and $20 \%$ of the neurons in S1 and S2, respectively. The absolute amounts of information that we obtained were comparable to the numbers that have been reported by several groups working with visual cortical neurons (Richmond and Optican, 1990; Gawne and Richmond, 1993; Gochin et al., 1994; Tovee et al., 1995). These studies were based on various kinds of visual stimuli that were effective at driving the tested neural populations, just as flutter was in our case. The agreement between these information values and ours might be attributable to network properties that are common to widely different cortical areas. Second, rate modulations in S1 and S2 conveyed similar amounts of information in periodic and aperiodic conditions, which raises the possibility that the same code for mean stimulus frequency is used in the two tasks. Third, the firing rate in both areas was modulated by the behavioral relevance of the stimuli, and we estimated the net impact of this contextual modulation: the numbers of spikes fired at a given frequency were more reliable when the animals were actively discriminating than when the stimuli were applied passively and, presumably, were ignored (Figs. 4a,c, 5a,c). On average, then, the firing rate provided a clearer, more reliable signal encoding stimulus frequency when this quantity was relevant to behavior. Last, we also found that the fluctuations in firing rate of some neurons were significantly correlated with the animals' psychophysical performance on a single trial basis, suggesting that a few additional spikes fired by a single cell may have a detectable impact on discrimination performance, even in the case of a primary sensory neuron (Leopold and Logothetis, 1996).

Although not conclusive in terms of the specific question we pursued, the experiments with periodic stimuli revealed a number of interesting facts about the timing of evoked spikes in the somatosensory cortices. First, as found earlier, periodicity was extremely high in S1 (Fig. 2f,h,i), and presumably it is even higher in primary afferents (Talbot et al., 1968; Vallbo, 1995), but periodicity diminished appreciably from area 3 b to area 1 (Fig. $2 i$ ) and almost vanished in S2, suggesting that it is limited to early cortical representations. In view of this and of the presence of significant rate modulations already at the level of S1, the question that arises is whether the strikingly regular temporal structure of S1 spikes is somehow exploited independently from variations in mean firing rate to compute or encode stimulus frequency. The second finding regarding timing was that the degree of periodicity in S1 was also affected by the behavioral relevance of the stimuli: evoked spikes were more phase-locked to the stimulus during active discrimination than during passive stimulation (Fig. $4 b, d$ ). Not surprisingly, this effect was not seen in S2 (Fig. $5 b, d$ ), where periodicity was much less prominent, although other timing effects have recently been described in this area (Steinmetz et al., 2000). Last, we found no relationship between variations in periodicity and psychophysical performance in single trials. None of the measures of periodicity that we tested exhibited significant covariations with behavior (Figs. $8 c, d$, and $9 c, d$ ). Clearly, analyses of this sort can only reveal subtle effects, especially when primary sensory neurons are concerned, but significant covariations between firing rate and performance were indeed found in S1 (Fig. $8 a, b$ ). This suggests that firing rate may have a larger weight in determining the neural code for stimulus frequency than the periodic alignment of spikes.

Coding mechanisms based exclusively on periodicity appear to be too rigid, as illustrated by the experiments with aperiodic stimulation: periodicity in S1 activity could not encode mean frequency during stimulation with aperiodic patterns, in the sense that mean frequency could not be read out from a Fourier decomposition of the evoked S1 responses, but temporal integration in a wider sense cannot be ruled out by these results. One key question in the discrimination task performed by our monkeys is how neurons postsynaptic to S1 integrate their incoming inputs: are they are able to read out some of the temporal features of the evoked activity? Neurons downstream from S1 must respond to certain features in the temporal structure of S1 activity, and they account for the ability of human subjects to distinguish without difficulty between periodic and aperiodic stimuli. From the comparisons between $I_{\mathrm{RATE}}$ and $I_{\mathrm{AIBI}}$ it appears that such features need to be more sophisticated than plain bursts of spikes from single neurons, but other schemes are possible. We also found that the precise timing of S1 spikes, in relation to the periodic stimulation pulses, was not appreciably correlated with psychophysical performance, but there might be particular time scales or temporally sensitive processes for which variations in S1 spike timing do have an impact in postsynaptic activity and in the code for flutter frequency, and the present experimental design or the analytic tools that we used may have been insensitive to those time scales. In the present task, the monkeys were not required to extract any features from the temporal structure of the stimuli other than the mean frequency, but the results - in particular the evoked activity in S2-might be different in tasks that do require such detailed temporal analysis.

Evidently, single cell recordings also have limitations; possible neural codes based on coordinated spike timing across multiple neurons have been reported (O'Keefe and Recce, 1993; DeCharms and Merzenich, 1995; Riehle et al., 1997; Dan et al., 1998). The following scenario, for instance, could apply to our task: the rate of synchronized spikes from two neurons might vary independently of the individual firing rates, providing additional information about the stimulus that cannot be extracted if synchronous and nonsynchronous spikes are considered equal and are lumped together to compute the firing rates (Dan et al., 1998). This is just one possible coding scheme based on temporal relationships between spikes. In 
general, these codes may be subtle, and testing whether they have a functional impact may be difficult. Even if they are present, neurons may or may not combine them with firing rate modulations to encode messages efficiently. To get a better understanding of neural coding in the cortex, what needs to be assessed is the effective contribution of all of these mechanisms to behavior.

\section{REFERENCES}

Abbott LF, Dayan P (1999) The effect of correlated activity on the accuracy of a population code. Neural Comput 11:91-101.

Abbott LF, Rolls ET, Tovee MJ (1996) Representational capacity of face coding in monkeys. Cereb Cortex 6:498-505.

Ahissar E (1998) Temporal-code to rate-code conversion by neuronal phase-locked loops. Neural Comput 10:597-650.

Ahissar E, Vaadia E (1990) Oscillatory activity of single units in a somatosensory cortex of an awake monkey and their possible role in texture analysis. Proc Natl Acad Sci USA 87:8935-8939.

Cariani PA, Delgutte B (1996) Neural correlates of the pitch of complex tones. I. Pitch and pitch salience. J Neurophysiol 76:1698-1716.

Chubbuck JK (1966) Small motion biological stimulator. Appl Digest 5:1319-1341.

Cover TM, Thomas JA (1991) Elements of information theory. New York: Wiley.

Dan Y, Alonso JM, Usrey WM, Reid RC (1998) Coding of visual information by precisely correlated spikes in the lateral geniculate nucleus. Nat Neurosci 1:501-507.

DeCharms RC, Merzenich MM (1995) Primary cortical representation of sounds by the coordination of action potential timing. Nature 381:610-613.

Efron B (1982) The jacknife, the bootstrap and other resampling plans. Philadelphia: Society for Industrial and Applied Mathematics.

Fitzgerald PJ, Lane JW, Yoshioka T, Nakama T, Hsiao SS (1999) Multidigit receptive field structures and orientation tuning properties of neurons in SII cortex of the awake monkey. Soc Neurosci Abstr 25:1684.

Gardner EP, Palmer CI, Hamalainen HA, Warren S (1992) Simulation of motion on the skin. V. Effect of stimulus temporal frequency on the representation of moving bar patterns in primary somatosensory cortex of monkeys. J Neurophysiol 67:37-63.

Gawne TJ, Richmond BJ (1993) How independent are the messages carried by adjacent inferior temporal neurons? J Neurosci 13:2758-2771.

Gochin PM, Colombo M, Dorfman GA, Gerstein GL, Gross CG (1994) Neural ensemble coding in inferior temporal cortex. J Neurophysiol 71:2325-2337.

Hernández A, Salinas E, García R, Romo R (1997) Discrimination in the sense of flutter: new psychophysical measurements in monkeys. J Neurosci 17:6391-6400.

Hsiao SS, Johnson KO, O'Shaughnessy DM (1993) Effects of selective attention of spatial form processing in monkey primary and secondary somatosensory cortex. J Neurophysiol 70:444-447.

Jones EG (1975) Lamination and differential distribution of the thalamic afferents within the sensory-motor cortex of the squirrel monkeys. J Comp Neurol 160:167-204.

Jones EG (1983) Lack of collateral thalamocortical projection to fields of the first somatic sensory cortex in monkeys. Exp Brain Res 52:375-384.

LaMotte RH, Mountcastle VB (1975) The capacities of humans and monkeys to discriminate between vibratory stimuli of different frequency and amplitude: a correlation between neural events and psychophysical measurements. J Neurophysiol 38:539-559.

LaMotte RH, Mountcastle VB (1979) Disorders in somesthesis following lesions of parietal lobe. J Neurophysiol 42:400-419.

Leopold DA, Logothetis NK (1996) Activity changes in early visual cortex reflect monkeys' percepts during binocular rivalry. Nature 379:549-553.

McAdams CJ, Maunsell JHR (1999) Effects of attention on orientation tuning functions of single neurons in macaque cortical area V4. J Neurosci 19:431-441.

McLurkin JW, Optican LM, Richmond BJ, Gawne TJ (1991) Concurrent processing and complexity of temporally encoded neuronal messages in visual perception. Science 253:675-677.

Mountcastle VB, Talbot WH, Darian-Smith I, Kornhuber HH (1967) Neural basis of the sense of flutter-vibration. Science 155:597-600.

Mountcastle VB, Talbot WH, Sakata H, Hyvärinen J (1969) Cortical neuronal mechanisms in flutter-vibration studied in unanesthetized monkeys. Neuronal periodicity and frequency discrimination. J Neurophysiol $32: 453-484$

Mountcastle VB, Steinmetz MA, Romo R (1990) Frequency discrimination in the sense of flutter: psychophysical measurements correlated with postcentral events in behaving monkeys. J Neurosci 10:3032-3044.

Mountcastle VB, Reitboek HJ, Poggio GF, Steinmetz MA (1991) Adaptation of the Reitboek method of multiple microelectrode recording to the neocortex of the waking monkey. J Neurosci Methods 36:77-84.
Ochoa J, Torebjörk E (1983) Sensations evoked by intraneural microstimulation of single mechanoreceptor units innervating the human hand. J Physiol (Lond) 342:633-654.

O'Keefe J, Recce ML (1993) Phase relationship between hippocampal place units and the EEG theta rhythm. Hippocampus 3:317-330.

Pons TP, Garraghty PE, Friedman DP, Mishkin M (1987) Physiological evidence for serial processing in somatosensory cortex. Science 237:417-420.

Pons TP, Garraghty PE, Mishkin M (1992) Serial and parallel processing of tactal information in somatosensory cortex of rhesus monkeys. J Neurophysiol 68:518-527.

Press WH, Teukolsky SA, Vetterling WT, Flannery BP (1992) Numerical recipes in C. Cambridge: Cambridge UP.

Recanzone GH, Merzenich MM, Schreiner CE (1992) Changes in the distributed temporal response properties of S1 cortical neurons reflect improvements in performance on a temporally based tactile discrimination task. J Neurophysiol 67:1071-1091.

Richmond BJ, Optican L (1990) Temporal encoding of two-dimensional patterns by single units in primate primary visual cortex. II. Information transmission. J Neurophysiol 64:370-380.

Riehle A, Grün S, Diesemann M, Aertsen A (1997) Spike synchronization and rate modulation differentially involved in motor cortical function. Science 278:1950-1953.

Romo R, Merchant H, Zainos A, Hernández A (1996) Categorization of somaesthetic stimuli: sensorimotor performance and neuronal activity in primary somatic sensory cortex of awake monkeys. NeuroReport 7:1273-1279.

Romo R, Hernández A, Zainos A, Salinas E (1998) Somatosensory discrimination based on cortical microstimulation. Nature 392:387-390.

Romo R, Brody CD, Hernández A, Lemus L (1999) Neuronal correlates of parametric working memory in the prefrontal cortex. Nature 399:470-473.

Salinas E, Romo R (1998) Conversion of sensory signals into motor commands in primary motor cortex. J Neurosci 18:499-511.

Shadlen MN, Newsome WT (1994) Noise, neural codes and cortical organization. Curr Opin Neurobiol 4:569-579.

Shadlen MN, Newsome WT (1998) The variable discharge of cortical neurons: implications for connectivity, computation and information coding. J Neurosci 18:3870-3896.

Siegel S, Castellan NJ (1988) Nonparametric statistics for the behavioral sciences. New York: McGraw-Hill.

Sinclair RJ, Burton H (1991) Neuronal activity in the primary somatosensory cortex in monkeys (Macaca mulatta) during active touch of textured surface gratings: responses to groove width, applied force, and velocity of motion. J Neurophysiol 66:153-169.

Sinclair RJ, Burton H (1993) Neuronal activity in the second somatosensory cortex of monkeys (Macaca mulatta) during active touch of gratings. J Neurophysiol 70:331-350.

Singer W, Gray CM (1995) Visual feature integration and the temporal correlation hypothesis. Annu Rev Neurosci 18:555-586.

Softky WR, Koch C (1993) The highly irregular firing of cortical cells is inconsistent with temporal integration of random EPSPs. J Neurosci 13:334-350.

Steinmetz PN, Roy A, Fitzgerald PJ, Hsiao SS, Johnson KO, Niebur E (2000) Attention modulates synchronized neuronal firing in primate somatosensory cortex. Nature 404:187-190.

Talbot WH, Darian-Smith I, Kornhuber HH, Mountcastle VB (1968) The sense of flutter vibration: comparison of the human capacity with response patterns of mechanoreceptive afferents from the monkey hand. J Neurophysiol 31:301-334.

Tovee MJ, Rolls ET, Treves A, Bellis RP (1995) Information encoding and the responses of single neurons in the primate temporal visual cortex. J Neurophysiol 70:640-654.

Treue S, Martínez-Trujillo JC (1999) Feature-based attention influences motion processing gain in macaque visual cortex. Nature 399:575-579.

Treves A, Panzeri S (1995) The upward bias in measures of information derived from limited data samples. Neural Comput 7:399-407.

Vallbo AB (1995) Single-afferent neurons and somatic sensation in humans. In: The cognitive neurosciences (Gazzaniga MS, ed), pp 237-252. Cambridge, MA: MIT.

Wickersham I, Groh JM (1998) Electrically evoking sensory experience. Curr Biol 8:R412-R414.

Zainos A, Merchant H, Hernández A, Salinas E, Romo R (1997) Role of primary somatic sensory cortex in the categorization of tactile stimuli: effects of lesions. Exp Brain Res 115:357-360.

Zhou YD, Fuster JM (1996) Mnemonic neuronal activity in somatosensory cortex. Proc Natl Acad Sci USA 93:10533-10537.

Zhou YD, Fuster JM (1997) Neuronal activity of somatosensory cortex in a cross-modal (visuo-haptic) memory task. Exp Brain Res 116:551-555.

Zohary E, Shadlen MN, Newsome WT (1994) Correlated neuronal discharge rate and its implications for psychophysical performance. Nature 370:140-143. 